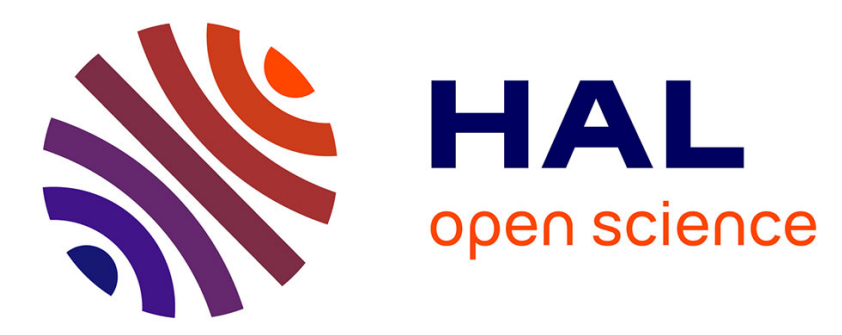

\title{
Competitive energy transfer between a two degree-of-freedom dynamic system and an absorber with essential nonlinearity
}

\author{
Thanh Tung Pham, Stéphane Pernot, Claude Henri Lamarque
}

\section{- To cite this version:}

Thanh Tung Pham, Stéphane Pernot, Claude Henri Lamarque. Competitive energy transfer between a two degree-of-freedom dynamic system and an absorber with essential nonlinearity. Nonlinear Dynamics, 2010, 62 (3), pp.573-592. 10.1007/s11071-010-9745-z . hal-00600462

\section{HAL Id: hal-00600462 \\ https://hal.science/hal-00600462}

Submitted on 15 Jun 2011

HAL is a multi-disciplinary open access archive for the deposit and dissemination of scientific research documents, whether they are published or not. The documents may come from teaching and research institutions in France or abroad, or from public or private research centers.
L'archive ouverte pluridisciplinaire HAL, est destinée au dépôt et à la diffusion de documents scientifiques de niveau recherche, publiés ou non, émanant des établissements d'enseignement et de recherche français ou étrangers, des laboratoires publics ou privés. 


\title{
COMPETITIVE ENERGY TRANSFER BETWEEN A TWO DEGREE-OF-FREEDOM DYNAMIC SYSTEM AND AN ABSORBER WITH ESSENTIAL NONLINEARITY
}

Thanh Tung PHAM

Stéphane PERNOT*

Claude Henri LAMARQUE

Département Génie Civil et Bâtiment URA-CNRS 1652

École Nationale des Travaux Publics de l'État

rue Maurice Audin, 69518 Vaulx-en-Velin Cedex, France

\begin{abstract}
The background of this work is related to passive vibration control of a two degree-of-freedom master system attached to an essentially nonlinear slave absorber aimed to attenuate vibrations by irreversibly transferring energy to a localized nonlinear normal mode ${ }^{1}$. Such nonlinear absorber which has no preferential frequency is theoretically able to capture several nonlinear resonances. The main purpose is here to bring an insight in what is actually going on when two linear modes are in competition for energy transfer. An original asymptotic analysis using two small parameters enables to build a scenario that improves the understanding of resonance mechanisms and to forecast which mode will be first attenuated by means of energy transfer. Numerical benchmark simulations corroborate the reliability of obtained scenario.

Key words: Passive vibration attenuation, energy transfer, nonlinear absorber, nonlinear normal modes, asymptotic analysis.
\end{abstract}

\section{Introduction}

The concept of targeted energy transfer has been extensively studied and it proved to be very efficient to mitigate vibration in dynamic systems. Energy pumping mechanism consists in irreversibly transferring vibratory energy from a master system to an essentially nonlinear coupled auxiliary system - namely the nonlinear energy sink NES - by triggering resonances between related nonlinear normal modes [? ? ]

\footnotetext{
${ }^{1}$ Nonlinear normal modes should be considered here according to the definition from Rosenberg [29]

* Corresponding author

Email addresses: stephane.pernot@entpe.fr (Stéphane PERNOT)

Preprint submitted to Nonlinear Dynamics
}

June 1, 2010 
followed by a bifurcation jump as explained in [? 1]. This resonance phenomenon is irreversible because of the existence of dissipation due for instance to internal viscous damping $[2,3,4]$ which means that vibration then remain trapped or localized into the NES slave system. Yet, energy transfer is permitted only when an intrinsic energy threshold is went through by master system.

Substantial theoretical and experimental work has also been achieved to bring a better understanding of energy transfer mechanisms and to validate the feasibility of targeted energy transfer. In [6], Vakakis and al investigated the feasibility and efficiency of nonlinear localization for isolating structures from earthquakeinduced motions. In [7, 8, 9, 10], McFarland and al developed an experimental setup to assess the reliability of a nonlinear energy sink device to mitigate the vibrations of a single degree-of-freedom system. Still in the context of mechanical engineering, Pernot and al and Gourdon and al led similar experiments [11, 12, 13, $14,15]$ with a four storey building model on top of which several NES absorbers were mounted to trigger irreversible energy transfer from the master modes towards the NESs. Configurations with NESs assembled in series were also considered to speed up modal localization. In [16, 17], Gendelman and al and Vakakis and al investigated from a theoretical point of view a system of multiple linear coupled oscillators involving an essentially nonlinear attachment. These studies highlight many advantages of the concept of modal localization to trigger targeted resonance captures with one of the system's mode. The concept was soon extended to other fields of research. Manevitch and al [18] used a strong nonlinear attachment to control the vibrations of semi-infinite chains of particules. In [19], Vakakis and al used NESs for shock isolation purposes. In [20], energy transfer was seek to attenuate transient vibrations of a dispersive elastic wave guide.

Further academic works $[21,22,23]$ soon focused on the ability of essential nonlinear absorbers to attenuate multiple resonance captures simultaneously or in cascade. In practice, a master system model with several degrees of freedom coupled with an auxiliary nonlinear system has been considered in [21] by proposing scenarios of isolated resonances and energy transfer within these scenarios. The analytical treatment has been made with the assumption of considering a single frequency of NES in each time interval and the numerical verification indicates the threshold energy necessary for targeted energy transfer. Latest developments aimed to study typical energy transfer mechanisms encountered for instance in the case of nonlinear systems involving multiple states of equilibrium [24] or in strongly nonlinear systems featuring nonhomogeneous nonlinearity [25]. Harmonically forced quasi-periodic regimes provoked by energy transfer were also studied in $[26,27]$. A simplified theoretical understanding of nonlinear localization is finally proposed in [28] where the class of considered attachment is no longer restricted to polynomial like nonlinearity but also to non polynomial one.

Contrary to standard linear or weakly nonlinear tuned mass absorbers that are designed to attenuate a single frequency stationary regime of master system, NES systems have no preferential frequency and are also fitted to work with transient vibration regimes. They are therefore theoretically able to simultaneously 
attenuate several modes of a master system as long as feeding energy is sufficient to maintain an active energy transfer. In practical experiments, however, simultaneous energy transfer has to the best of authors knowledge never been observed. As an attempt of explanation, the answer is maybe to be sought by investigating the bassins of attraction of underlying nonlinear localized modes. Hence, let us consider the following ersatz stating that if multiple modal localization are likely to occur then a precedence mechanism must prevail.

Present work also aims to accurately understand energy transfer mechanisms likely to occur in the mitigation of a general linear two degree-of-freedom system attached to a NES device. The main purpose is first to understand what is going on when two nonlinear modal localizations compete against each other and second to build scenarios eventually permitting to forecast which resonance will be triggered first during transient regime - the idealistic case corresponding to large amplitude vibration localized in the NES absorber and small amplitude oscillation in the master system. Present paper is organized as follows: section 2 introduces the methodology upon the academic instance of a two degree-of-freedom master system attached to a purely cubic nonlinear oscillator. It is noticeable that case study is different from the one investigated in [21] for nonlinear attachment is not connected to the ground. After re-normalizing equations of motion in the linear modal base, an original asymptotic expansion with two independent scales is performed on previous equations. A superposition principle of nonlinear normal modes is introduced up to the first scale order to split motion into two new independent coordinates, the latter of which characterizing the amount of energy that is transferred between each master degree-of-freedom and the NES device. Manevitch's complexification method [5] is then used to investigate the dynamics response of related nonlinear normal modes close to a double one to one resonance. A multiple scale technique is finally applied to exhibit reduced nonlinear normal modes. Section 3 is dedicated to the study of targeted energy transfers (TET) that enable to forecast the transient dynamic response of the two dofs + NES system. The peculiar case of well separated master modes is investigated and allow to furnish an analytical trend of energy transfer versus time that is similar with results obtained by O. Gendelman in [22]. Going back to the general case, multiple scenarios are introduced to forecast energy transfers in the academic system - the approach being to slave one scale to the other in reduced equations of motion - Numerical evidences introduced in section 4 allow to corroborate the reliability of previous energy pumping scenarios. Conclusions are finally drawn and direction of prospective research works is given.

\section{Two-scale asymptotic expansion of an academic model}

The energy pumping phenomenon has been widely investigated in $[6,19]$ for single degree-of-freedom master systems attached to slave mass by means of an essential nonlinear stiffness. Main features are a oneway and irreversible transfer of master system vibrations towards the NES device where energy is localized 


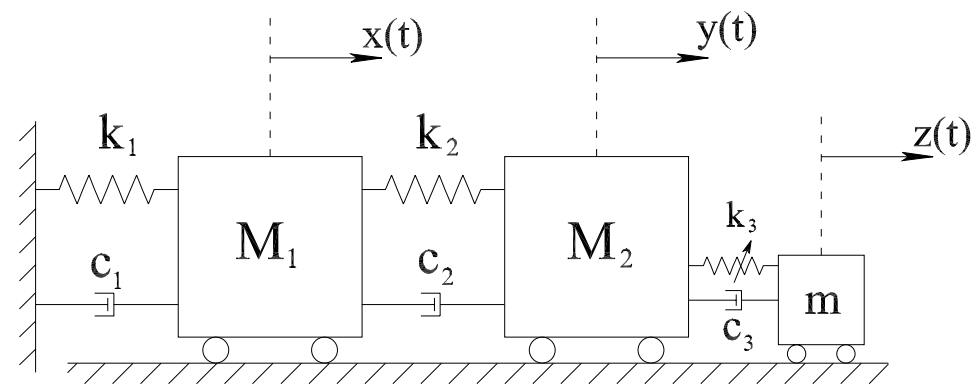

Figure 1: Two dofs + NES system.

before vanishing thanks to the occurrence of damping [4, 2]. Hence nonlinear attachment is at the roots of a one to one resonance capture responsible for energy pumping phenomenon. Present study now aims to investigate mechanisms of energy transfer when master system includes two degree-of-freedom as depicted in Fig. 1.

\subsection{Two-dofs master system coupled with a NES}

Let us consider the following academic spring-mass-damper system obtained for instance by applying reduction theory and connected to a nonlinear spring/mass system namely the NES device. Applying Newton's second law of motion, it yields the following equations of motion written in the physical basis:

$$
\begin{cases}M_{1} \ddot{x}+c_{1} \dot{x}+c_{2}(\dot{x}-\dot{y})+k_{1} x+k_{2}(x-y) & =0 \\ M_{2} \ddot{y}+c_{2}(\dot{y}-\dot{x})+k_{2}(y-x)+c_{3}(\dot{y}-\dot{z})+k_{3}(y-z)^{3} & =0 \\ m \ddot{z}+c_{3}(\dot{z}-\dot{y})+k_{3}(z-y)^{3} & =0\end{cases}
$$

where $x, y$ and $z$ respectively stand for the displacement of the first, the second mass and the NES absorber. $k_{3}$ refers to a purely cubic nonlinear stiffness which means that related restoring force is nonlinear proportional with the internal displacement $(z-y)$ and $\dot{x}$ is the derivative of variable $x$ with respect to time $t$.

Previous set of equations (1) may be rewritten in matrix form

$$
\left\{\begin{array}{cc}
\underline{\underline{M}} \underline{\ddot{X}}+\underline{\underline{C}} \underline{\dot{X}}+\underline{\underline{K}} \underline{X}+\left[\begin{array}{c}
0 \\
c_{3}(\dot{y}-\dot{z})+k_{3}(y-z)^{3}
\end{array}\right] & =\left[\begin{array}{l}
0 \\
0
\end{array}\right] \\
m \ddot{z}+c_{3}(\dot{z}-\dot{y})+k_{3}(z-y)^{3} & =0
\end{array}\right.
$$

involving mass matrix $\underline{\underline{M}}=\left[\begin{array}{cc}M_{1} & 0 \\ 0 & M_{2}\end{array}\right]$, stiffness matrix $\underline{\underline{K}}=\left[\begin{array}{rr}k_{1}+k_{2} & -k_{2} \\ -k_{2} & k_{1}\end{array}\right]$, damping matrix $\underline{\underline{C}}=\left[\begin{array}{rr}c_{1}+c_{2} & -c_{2} \\ -c_{2} & c_{1}\end{array}\right]$ and displacement vector $\underline{X}=\left[\begin{array}{l}x \\ y\end{array}\right]$ 


\subsection{Model written in the modal basis}

Assuming that nonlinear coupling does not change eigenmodes of master system so far, equations of motion (2) may be spanned in the modal basis by introducing the following modal change of coordinates:

$$
\underline{X}=\underline{\underline{\Phi}} \cdot \underline{q}
$$

involving mode shape vectors $\underline{\underline{\Phi}}=\left[\begin{array}{ll}\underline{\phi}_{1} & \underline{\phi}_{2}\end{array}\right]=\left[\begin{array}{cc}\phi_{11}, & \phi_{12} \\ \phi_{12}, & \phi_{22}\end{array}\right]$ and modal coordinates $\underline{q}=\left[\begin{array}{c}q_{1} \\ q_{2}\end{array}\right]$

All simplifications done, one finally obtains:

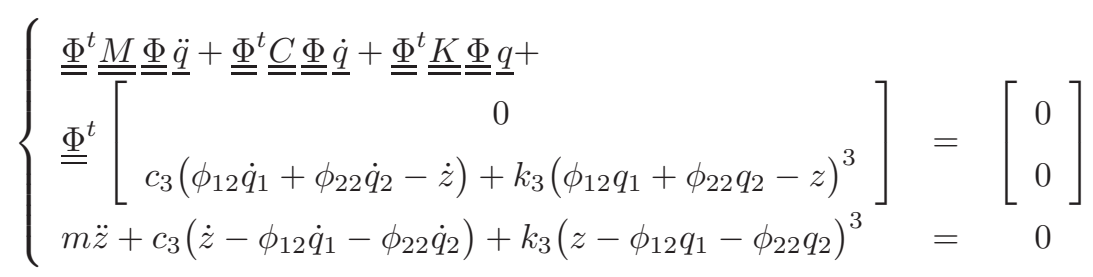

Orthogonal properties of eigenmodes with respect to mass $M$ and stiffness $K$ matrices then enable to partly uncouple equations (4) by equating

$$
\underline{\underline{\Phi}}^{t} \underline{\underline{M}} \underline{\underline{\Phi}}=\left[\begin{array}{cc}
M_{11} & 0 \\
0 & M_{22}
\end{array}\right]
$$

and

$$
\underline{\underline{\Phi}}^{t} \underline{\underline{K}} \underline{\underline{\Phi}}=\left[\begin{array}{cc}
k_{11} & 0 \\
0 & k_{22}
\end{array}\right]
$$

and damping matrix

$$
\underline{\underline{\Phi}}^{t} \underline{\underline{C}} \underline{\underline{\Phi}}=\left[\begin{array}{ll}
c_{11} & c_{21} \\
c_{12} & c_{22}
\end{array}\right]
$$

Equations of motion consequently become

$$
\begin{cases}M_{11} \ddot{q}_{1}+c_{11} \dot{q}_{1}+c_{21} \dot{q}_{2}+k_{11} q_{1}+\phi_{12} c_{3}\left(\phi_{12} \dot{q}_{1}+\phi_{22} \dot{q}_{2}-\dot{z}\right)+\phi_{12} k_{3}\left(\phi_{12} q_{1}+\phi_{22} q_{2}-z\right)^{3} & =0 \\ M_{22} \ddot{q}_{2}+c_{12} \dot{q}_{1}+c_{22} \dot{q}_{2}+k_{22} q_{2}+\phi_{22} c_{3}\left(\phi_{12} \dot{q}_{1}+\phi_{22} \dot{q}_{2}-\dot{z}\right)+\phi_{22} k_{3}\left(\phi_{12} q_{1}+\phi_{22} q_{2}-z\right)^{3} & =0 \\ m \ddot{z}+c_{3}\left(\dot{z}-\phi_{12} \dot{q}_{1}-\phi_{22} \dot{q}_{2}\right)+k_{3}\left(z-\phi_{12} q_{1}-\phi_{22} q_{2}\right)^{3} & =0\end{cases}
$$

\subsection{Non-dimensional equations of motion}

Introducing renormalization parameters $y_{1}=\phi_{12} q_{1}, y_{2}=\phi_{22} q_{2}, \omega_{1}^{2}=\frac{k_{11}}{M_{11}}, \omega_{2}^{2}=\frac{k_{22}}{M_{22}}, \lambda=\frac{c_{3}}{m}, K=\frac{k_{3}}{m}$, $\epsilon_{1}=\frac{\phi_{12}^{2} m}{M_{11}}, \epsilon_{2}=\frac{\phi_{22}^{2} m}{M_{22}}, \epsilon_{1} \lambda_{1}=\frac{c_{11}}{M_{11}}, \epsilon_{2} \lambda_{2}=\frac{c_{22}}{M_{22}}, \epsilon_{1} \lambda_{21}=\frac{\phi_{12}}{\phi_{22}} \frac{c_{21}}{M_{11}}, \epsilon_{2} \lambda_{12}=\frac{\phi_{22}}{\phi_{12}} \frac{c_{12}}{M_{22}}$, into equation (8), one obtains the following two-scale equations of motion:

$$
\begin{cases}\ddot{y}_{1}+\epsilon_{1} \lambda_{1} \dot{y}_{1}+\epsilon_{1} \lambda_{21} \dot{y}_{2}+\omega_{1}^{2} y_{1}+\epsilon_{1} \lambda\left(\dot{y}_{1}+\dot{y}_{2}-\dot{z}\right)+\epsilon_{1} K\left(y_{1}+y_{2}-z\right)^{3} & =0 \\ \ddot{y}_{2}+\epsilon_{2} \lambda_{12} \dot{y}_{1}+\epsilon_{2} \lambda_{2} \dot{y}_{2}+\omega_{2}^{2} y_{2}+\epsilon_{2} \lambda\left(\dot{y}_{1}+\dot{y}_{2}-\dot{z}\right)+\epsilon_{2} K\left(y_{1}+y_{2}-z\right)^{3} & =0 \\ \ddot{z}+\lambda\left(\dot{z}-\dot{y}_{1}-\dot{y}_{2}\right)+K\left(z-y_{1}-y_{2}\right)_{5}^{3} & =0\end{cases}
$$


involving two small scale parameters $\epsilon_{1} \ll 1$ and $\epsilon_{2} \ll 1$ homogeneous to mass ratios.

Original problem (1) is hence transformed into a set of 3 weakly coupled differential equations. Modal coordinates $y_{1}$ (resp. $y_{2}$ ) are weakly coupled with $y_{2}$ and $z$ (resp. with $y_{1}$ and $z$ ) in the first two equations while at the same time NES coordinate $z$ remains strongly connected to $y_{1}$ and $y_{2}$ in the last equation. In what follows, primary structure and NES device are assumed to be initially at rest. An impulse is then instantaneously exciting master coordinates $y_{1}$ and $y_{2}$ which is similar to consider the free oscillation response of master + NES system under some initial conditions given by:

$$
y_{1}(t=0)=y_{2}(t=0)=z(t=0)=0, \frac{d y_{1}}{d t}=C_{I}, \frac{d y_{2}}{d t}=C_{I I}, \frac{d z}{d t}=0
$$

\subsection{Splitting of NES response}

Truncating two-scale series expansion of equations (9) at order $\epsilon_{1}^{0}$ and $\epsilon_{2}^{0}$ leads to a set of equations

$$
\left\{\begin{array}{lll}
\ddot{y}_{1}+\omega_{1}^{2} y_{1} & =0 \\
\ddot{y}_{2}+\omega_{2}^{2} y_{2} & =0 \\
\ddot{z}+\lambda\left(\dot{z}-\dot{y}_{1}-\dot{y}_{2}\right)+K\left(z-y_{1}-y_{2}\right)^{3} & =0
\end{array}\right.
$$

whose solutions $y_{1}$ and $y_{2}$ are completely uncoupled from NES response $z$. As a result, $y_{1}$ and $y_{2}$ are pure oscillations vibrating at frequencies $\omega_{1}$ and $\omega_{2}$ respectively. Because the motion of $z$ is dominated by components featuring $y_{1}$ and $y_{2}$, it is reasonable to assume that $z$ is mainly divided into two components $z_{1}$ and $z_{2}$ themselves vibrating at frequencies $\omega_{1}$ and $\omega_{2}$. A splitting $z=z_{1}+z_{2}$ is also introduced into equations (9) to account for this notice:

$$
\begin{cases}\ddot{y}_{1}+\epsilon_{1} \lambda_{1} \dot{y}_{1}+\epsilon_{1} \lambda_{21} \dot{y}_{2}+\omega_{1}^{2} y_{1}+\epsilon_{1} \lambda\left(\dot{y}_{1}-\dot{z}_{1}+\dot{y}_{2}-\dot{z}_{2}\right)+\epsilon_{1} K\left(y_{1}-z_{1}+y_{2}-z_{2}\right)^{3} & =0 \\ \ddot{y}_{2}+\epsilon_{2} \lambda_{12} \dot{y}_{1}+\epsilon_{2} \lambda_{2} \dot{y}_{2}+\omega_{2}^{2} y_{2}+\epsilon_{2} \lambda\left(\dot{y}_{1}-\dot{z}_{1}+\dot{y}_{2}-\dot{z}_{2}\right)+\epsilon_{2} K\left(y_{1}-z_{1}+y_{2}-z_{2}\right)^{3} & =0 \\ \ddot{z}_{1}+\ddot{z}_{2}+\lambda\left(\dot{z}_{1}-\dot{y}_{1}+\dot{z}_{2}-\dot{y}_{2}\right)+K\left(z_{1}-y_{1}+z_{2}-y_{2}\right)^{3} & =0\end{cases}
$$

A new change of variables:

$$
\begin{cases}u_{1}=y_{1}+\epsilon_{1} z_{1}, & v_{1}=y_{1}-z_{1} \\ u_{2}=y_{2}+\epsilon_{2} z_{2}, & v_{2}=y_{2}-z_{2}\end{cases}
$$

that exhibits the displacement of the center of inertia and the internal displacement of both modes 1 and 2 with respect to the NES is introduced and leads to equations:

$$
\left\{\begin{array}{l}
\frac{\ddot{u}_{1}+\epsilon_{1} \ddot{v}_{1}}{1+\epsilon_{1}}+\epsilon_{1} \lambda_{1} \frac{\dot{u}_{1}+\epsilon_{1} \dot{v}_{1}}{1+\epsilon_{1}}+\epsilon_{1} \lambda_{21} \frac{\dot{u}_{2}+\epsilon_{2} \dot{v}_{2}}{1+\epsilon_{2}}+\omega_{1}^{2} \frac{u_{1}+\epsilon_{1} v_{1}}{1+\epsilon_{1}}+\epsilon_{1} \lambda\left(\dot{v}_{1}+\dot{v}_{2}\right)+\epsilon_{1} K\left(v_{1}+v_{2}\right)^{3}=0 \\
\frac{\ddot{u}_{2}+\epsilon_{2} \ddot{v}_{2}}{1+\epsilon_{2}}+\epsilon_{2} \lambda_{2} \frac{\dot{u}_{2}+\epsilon_{2} \dot{v}_{2}}{1+\epsilon_{2}}+\epsilon_{2} \lambda_{12} \frac{\dot{u}_{1}+\epsilon_{1} \dot{v}_{1}}{1+\epsilon_{1}}+\omega_{2}^{2} \frac{u_{2}+\epsilon_{2} v_{2}}{1+\epsilon_{2}}+\epsilon_{2} \lambda\left(\dot{v}_{1}+\dot{v}_{2}\right)+\epsilon_{2} K\left(v_{1}+v_{2}\right)^{3}=0 \\
\frac{\ddot{u}_{1}-\ddot{v}_{1}}{1+\epsilon_{1}}+\frac{\ddot{u}_{2}-\ddot{v}_{2}}{1+\epsilon_{2}}-\lambda\left(\dot{v}_{1}+\dot{v}_{2}\right)-K\left(v_{1}+v_{2}\right)^{3} \\
=0
\end{array}\right.
$$




\subsection{Complexified problem}

Manevitch's complexification technique [? ] is used to highlight low dynamics of studied system from fast oscillating response. Fast components also appear to match modal frequencies $\omega_{1}$ and $\omega_{2}$ modulated by slow changing amplitudes $\left(\varphi_{i}\right)_{i=1 \cdot 4}$.

By inserting complex change of variables written as:

$$
\left\{\begin{aligned}
\varphi_{1} e^{i \omega_{1} t} & =\frac{\dot{u}_{1}}{\omega_{1}}+i u_{1}, & \varphi_{2} e^{i \omega_{1} t} & =\frac{\dot{v}_{1}}{\omega_{1}}+i v_{1} \\
\varphi_{3} e^{i \omega_{2} t} & =\frac{\dot{u}_{2}}{\omega_{2}}+i u_{2}, & \varphi_{4} e^{i \omega_{2} t} & =\frac{\dot{v}_{2}}{\omega_{2}}+i v_{2}
\end{aligned}\right.
$$

one similarly obtains:

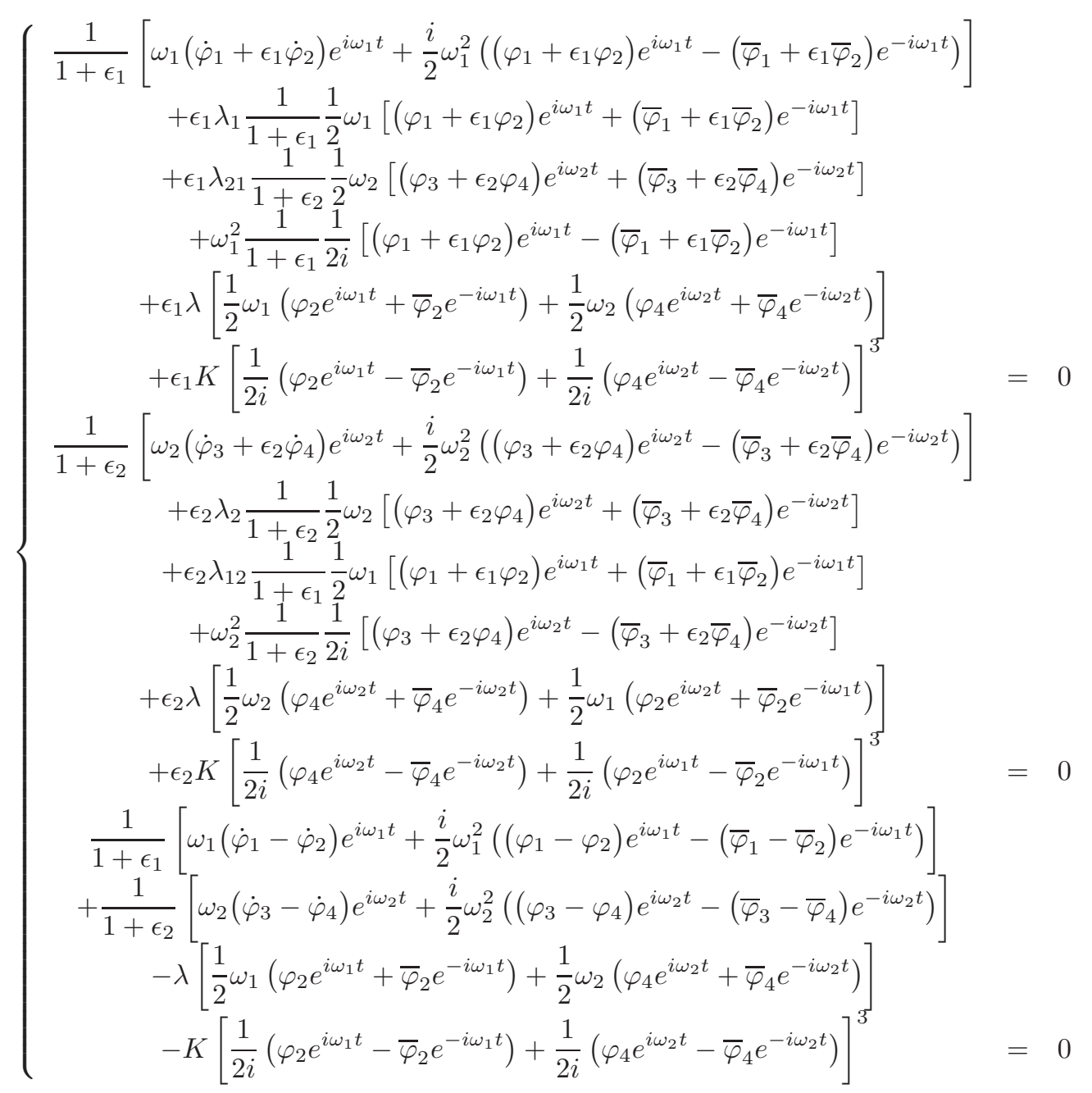

The main goal is now to cancel secular components in $\left(e^{i \omega_{j} t}\right)_{j=1,2}$ within related multiple scale approximations for only stationary and bounded solutions are seek.

An averaging method with resonant frequency $\omega_{1}$ is therefore applied on both first and third equations 
of system (16) which yields:

$$
\begin{cases}\omega_{1}\left(\dot{\varphi}_{1}+\epsilon_{1} \dot{\varphi}_{2}\right)-\frac{3}{4} i \epsilon_{1} K \varphi_{2}\left|\varphi_{4}\right|^{2}-\frac{3}{8} i \epsilon_{1} K \varphi_{2}\left|\varphi_{2}\right|^{2}+\frac{1}{2} \epsilon_{1} \lambda \omega_{1} \varphi_{2}+\frac{1}{2} \epsilon_{1} \lambda_{1} \omega_{1} \varphi_{1} & = \\ \left(1+\epsilon_{2}\right) \omega_{1}\left(\dot{\varphi}_{1}-\dot{\varphi}_{2}\right)+\frac{3}{4} i\left(1+\epsilon_{1}+\epsilon_{2}\right) K \varphi_{2}\left|\varphi_{4}\right|^{2}+\frac{1}{2} i\left(1+\epsilon_{2}\right) \omega_{1}^{2}\left(\varphi_{1}-\varphi_{2}\right) & \\ -\frac{1}{2}\left(1+\epsilon_{1}+\epsilon_{2}\right) \lambda \omega_{1} \varphi_{2}+\frac{3}{8} i\left(1+\epsilon_{1}+\epsilon_{2}\right) K \varphi_{2}\left|\varphi_{2}\right|^{2} & =0\end{cases}
$$

Repeating similar averaging technique with resonant frequency $\omega_{2}$ on second and third equations of system (16), it yields:

$$
\begin{cases}\omega_{2}\left(\dot{\varphi}_{3}+\epsilon_{2} \dot{\varphi}_{4}\right)-\frac{3}{4} i \epsilon_{2} K \varphi_{4}\left|\varphi_{2}\right|^{2}-\frac{3}{8} i \epsilon_{2} K \varphi_{4}\left|\varphi_{4}\right|^{2}+\frac{1}{2} \epsilon_{2} \lambda \omega_{2} \varphi_{4}+\frac{1}{2} \epsilon_{2} \lambda_{2} \omega_{2} \varphi_{3} & =0 \\ \left(1+\epsilon_{1}\right) \omega_{2}\left(\dot{\varphi}_{3}-\dot{\varphi}_{4}\right)+\frac{3}{4} i\left(1+\epsilon_{1}+\epsilon_{2}\right) K \varphi_{4}\left|\varphi_{2}\right|^{2}+\frac{1}{2} i\left(1+\epsilon_{1}\right) \omega_{2}^{2}\left(\varphi_{3}-\varphi_{4}\right) & \\ -\frac{1}{2}\left(1+\epsilon_{1}+\epsilon_{2}\right) \lambda \omega_{2} \varphi_{4}+\frac{3}{8} i\left(1+\epsilon_{1}+\epsilon_{2}\right) K \varphi_{4}\left|\varphi_{4}\right|^{2} & =0\end{cases}
$$

By isolating $\dot{\varphi}_{1}$ and $\dot{\varphi}_{2}$ components and by considering a taylor series expansion up to order $O\left(\epsilon_{i}\right)_{i=1,2}$, system (17) may be written as:

$$
\left\{\begin{array}{lll}
\left(1+\epsilon_{1}+\epsilon_{2}\right) \dot{\varphi}_{1}+\frac{1}{2} i \epsilon_{1} \omega_{1} \varphi_{1}+\frac{1}{2} \epsilon_{1} \lambda_{1} \varphi_{1}-\frac{1}{2} i \epsilon_{1} \omega_{1} \varphi_{2}+O\left(\epsilon_{1}^{2}, \epsilon_{2}^{2}, \epsilon_{1} \epsilon_{2}\right) & = \\
\omega_{1} \dot{\varphi}_{2}-\frac{3}{8} i K \varphi_{2}\left|\varphi_{2}\right|^{2}-\frac{1}{2} i \omega_{1}^{2} \varphi_{1}+\frac{1}{2} i \omega_{1}^{2} \varphi_{2}-\frac{3}{4} i K \varphi_{2}\left|\varphi_{4}\right|^{2}+\frac{1}{2} \lambda \omega_{1} \varphi_{2}+O\left(\epsilon_{1}, \epsilon_{2}\right) & =0
\end{array}\right.
$$

Doing similarly with $\dot{\varphi}_{3}$ and $\dot{\varphi}_{4}$ in equations (18), one obtains:

$$
\begin{cases}\left(1+\epsilon_{1}+\epsilon_{2}\right) \dot{\varphi}_{3}+\frac{1}{2} i \epsilon_{2} \omega_{2} \varphi_{3}+\frac{1}{2} \epsilon_{2} \lambda_{2} \varphi_{3}-\frac{1}{2} i \epsilon_{2} \omega_{2} \varphi_{4}+O\left(\epsilon_{1}^{2}, \epsilon_{2}^{2}, \epsilon_{1} \epsilon_{2}\right) & =0 \\ \omega_{2} \dot{\varphi}_{4}-\frac{3}{8} i K \varphi_{4}\left|\varphi_{4}\right|^{2}-\frac{1}{2} i \omega_{2}^{2} \varphi_{3}+\frac{1}{2} i \omega_{2}^{2} \varphi_{4}-\frac{3}{4} i K \varphi_{4}\left|\varphi_{2}\right|^{2}+\frac{1}{2} \lambda \omega_{2} \varphi_{4}+O\left(\epsilon_{1}, \epsilon_{2}\right) & =0\end{cases}
$$

It should be pointed out that such results only hold when following conditions are simultaneously valid:

$$
\begin{aligned}
\omega_{1} & \neq \omega_{2} \\
3 \omega_{1} & \neq \omega_{2}
\end{aligned}
$$

\subsection{Two-scale multiple scale analysis of complexified model}

The method of multiple scales is applied on problem (20) by considering $\epsilon_{1}$ and $\epsilon_{2}$ like independent scale parameters. Introducing related time scales $T_{i}=\epsilon_{1}^{i} t$ and $\tau_{i}=\epsilon_{2}^{i} t$, new time derivatives may alternatively be derived:

$$
\frac{d}{d t} \longrightarrow \sum_{i=0}^{\infty} \epsilon_{1}^{i} \frac{\partial}{\partial T_{i}}
$$

or

$$
\frac{d}{d t} \longrightarrow \sum_{i=0}^{\infty} \epsilon_{2}^{i} \frac{\partial}{\partial \tau_{i}}
$$

Expanding complex amplitudes $\left(\varphi_{i}\right)_{i=1, \cdots, 4}$ by considering following changes of coordinates:

$$
\left\{\begin{array}{lll}
\varphi_{1}(t)=\varphi_{1}\left(T_{0}, T_{1}\right), & \varphi_{2}(t)=\varphi_{2}\left(T_{0}, T_{1}\right) \\
\varphi_{3}(t)=\varphi_{3}\left(\tau_{0}, \tau_{1}\right), & \varphi_{4}(t)=\varphi_{4}\left(\tau_{0}, \tau_{1}\right)
\end{array}\right.
$$


Because the energy drop provoked by energy pumping phenomenon is much faster than the one due to system's natural damping, it is reasonable to assume that following re-scaling rule holds:

$$
\lambda_{1}=\epsilon_{1} \lambda_{10}, \quad \lambda_{2}=\epsilon_{2} \lambda_{20}
$$

to minimize the influence of master system damping versus energy transfer.

Equations (19) and (20) may hence be expanded in telescopic series featuring scales $\left(\epsilon_{i}^{j}\right)_{i, j}$. Isolating latter expressions up to the first scale orders, one finally obtains:

- Equations with respect to scale orders $\epsilon_{1}^{i}$

- order $\epsilon_{1}^{0}$ :

$$
\begin{aligned}
\frac{\partial \varphi_{1}}{\partial T_{0}} & =0 \\
\omega_{1} \frac{\partial \varphi_{2}}{\partial T_{0}}-\frac{3}{8} i K \varphi_{2}\left|\varphi_{2}\right|^{2}-\frac{1}{2} i \omega_{1}^{2} \varphi_{1}+\frac{1}{2} i \omega_{1}^{2} \varphi_{2}-\frac{3}{4} i K \varphi_{2}\left|\varphi_{4}\right|^{2}+\frac{1}{2} \lambda \omega_{1} \varphi_{2} & =0
\end{aligned}
$$

- order $\epsilon_{1}^{1}$ :

$$
\frac{\partial \varphi_{1}}{\partial T_{1}}+\left(1+\frac{\epsilon_{2}}{\epsilon_{1}}\right) \frac{\partial \varphi_{1}}{\partial T_{0}}+\frac{1}{2} i \omega_{1} \varphi_{1}-\frac{1}{2} i \omega_{1} \varphi_{2}=0
$$

- Equations with respect to scale orders $\epsilon_{2}^{i}$

$-\operatorname{order} \epsilon_{2}^{0}$ :

$$
\begin{aligned}
\frac{\partial \varphi_{3}}{\partial \tau_{0}} & =0 \\
\omega_{1} \frac{\partial \varphi_{4}}{\partial \tau_{0}}-\frac{3}{8} i K \varphi_{4}\left|\varphi_{4}\right|^{2}-\frac{1}{2} i \omega_{2}^{2} \varphi_{3}+\frac{1}{2} i \omega_{2}^{2} \varphi_{4}-\frac{3}{4} i K \varphi_{4}\left|\varphi_{2}\right|^{2}+\frac{1}{2} \lambda \omega_{2} \varphi_{4} & =0
\end{aligned}
$$

- order $\epsilon_{2}^{1}$ :

$$
\frac{\partial \varphi_{3}}{\partial \tau_{1}}+\left(1+\frac{\epsilon_{1}}{\epsilon_{2}}\right) \frac{\partial \varphi_{3}}{\partial \tau_{0}}+\frac{1}{2} i \omega_{2} \varphi_{3}-\frac{1}{2} i \omega_{2} \varphi_{4}=0
$$

From equations (27) and (30), amplitudes $\varphi_{1}$ and $\varphi_{3}$ happen to be independent of $T_{0}$ and $\tau_{0}$ respectively which gives:

$$
\begin{aligned}
& \varphi_{1}=\varphi_{1}\left(T_{1}\right) \\
& \varphi_{3}=\varphi_{3}\left(\tau_{1}\right)
\end{aligned}
$$

In addition, one may assume that

$$
\begin{aligned}
\lim _{T_{0} \rightarrow \infty} \frac{\partial \varphi_{2}}{\partial T_{0}} & =0 \\
\lim _{\tau_{0} \rightarrow \infty} \frac{\partial \varphi_{4}}{\partial \tau_{0}} & =0 \\
9 & =
\end{aligned}
$$


for master system oscillations are asymptotically damped.

Let us also introduce following definitions in what follows:

$$
\begin{aligned}
& \psi_{2}\left(T_{1}\right)=\lim _{T_{0} \rightarrow \infty} \varphi_{2}\left(T_{0}, T_{1}\right) \\
& \psi_{4}\left(\tau_{1}\right)=\lim _{\tau_{0} \rightarrow \infty} \varphi_{4}\left(\tau_{0}, \tau_{1}\right)
\end{aligned}
$$

\subsection{Nonlinear modes of reduced model}

Nonlinear modes are by the end solutions of a set of reduced equations of motion written with respect to two independent time scales $T_{1}$ and $\tau_{1}$.

From equations (28) and (29), one obtains:

$$
\begin{cases}\frac{1}{2} \lambda \omega_{1} \psi_{2}-\frac{3}{8} i K \psi_{2}\left|\psi_{2}\right|^{2}-\frac{1}{2} i \omega_{1}^{2} \varphi_{1}+\frac{1}{2} i \omega_{1}^{2} \psi_{2}-\frac{3}{4} i K \psi_{2}\left|\psi_{4}\right|^{2} & =0 \\ \frac{\partial \varphi_{1}}{\partial T_{1}}+\frac{1}{2} i \omega_{1} \varphi_{1}-\frac{1}{2} i \omega_{1} \psi_{2} & =0\end{cases}
$$

From equations (31) and (32), one obtains:

$$
\left\{\begin{array}{l}
\frac{1}{2} \lambda \omega_{2} \psi_{4}-\frac{3}{8} i K \psi_{4}\left|\psi_{4}\right|^{2}-\frac{1}{2} i \omega_{2}^{2} \varphi_{3}+\frac{1}{2} i \omega_{2}^{2} \psi_{4}-\frac{3}{4} i K \psi_{4}\left|\psi_{2}\right|^{2}=0 \\
\frac{\partial \varphi_{3}}{\partial \tau_{1}}+\frac{1}{2} i \omega_{2} \varphi_{3}-\frac{1}{2} i \omega_{2} \psi_{4}=0
\end{array}\right.
$$

It is noticeable that equations (39) involve contributions depending mainly on time scale $T_{1}$ - nonlinear component $-\frac{3}{4} i K \psi_{2}\left|\psi_{4}\right|^{2}$ excepted which also depends on time scale $\tau_{1}$ - Similar result hold for equations (40) with respect to time scale $\tau_{1}$. Hence, competitive energy transfers between the first or the second mode and the NES device are ruled by the occurrence of nonlinear coupling featuring time scales $\left(T_{1}, \tau_{1}\right)$. Next section aims to bring an insight in what is potentially going on by investigating several scenarios for energy transfer.

\section{Targeted energy transfers in the two dofs + NES system}

Multiple scenarios are introduced hereafter to forecast transient energy transfers likely to develop in the studied dynamical system including two degrees of freedom connected to a NES by means of an essentially nonlinear stiffness. The peculiar case of well separated master modes is investigated and allow to furnish an analytical trend of energy transfer versus time. A general approach is then extended by slaving one scale to another in previous reduced equations of motion.

\subsection{TET mechanism for well separated master modes}

Let's here consider the peculiar case $\epsilon_{1} \ll \epsilon_{2}$ which may be translated for instance into the following ersatz $\epsilon_{1}=\chi^{3}$ and $\epsilon_{2}=\chi^{2}$ with $\chi \ll 1$. Previous multi-scale expansions are not fundamentally modified 
by these underlying scale changes and one can exploit related sets of equations. It is also reasonable to additionally assume that when time scale $\tau_{1}$ begins to increase, time scale $T_{1}$ remains constant for a given duration.

Energy $\psi_{2}$ may hence be regarded as a constant mapping in the first equation of system (40) by writing:

$$
\left|\psi_{2}\right|^{2}=C_{1}
$$

where $C_{1}$ stands for the 'initial' energy ${ }^{2}$ embedded in the first differential mode.

After replacing statement (41) into equation (40) finally yields:

$$
\varphi_{3}=\left(1-\frac{3}{2} C_{1} \Omega_{2}\right) \psi_{4}-i \gamma_{2} \psi_{4}-\frac{3}{4} i \Omega_{2} \psi_{4}\left|\psi_{4}\right|^{2}
$$

featuring news parameters $\Omega_{2}=\frac{K}{\omega_{2}^{2}}$ and $\gamma_{2}=\frac{\lambda}{\omega_{2}}$.

In order to better understand energy transfer towards second differential mode, variable $\psi_{4}$ is transformed into following polar coordinates:

$$
\psi_{4}=N_{2} e^{i \delta_{2}}
$$

and equation (40) finally leads to the simplified expression:

$$
\frac{d Z_{2}}{d \tau_{1}}=-\frac{16 \omega_{2} \gamma_{2} Z_{2}}{27 \Omega_{2}^{2} Z_{2}^{2}-\left(48 \Omega_{2}-72 \Omega_{2}^{2} C_{1}\right) Z_{2}+16 \gamma_{2}^{2}+16+36 \Omega_{2}^{2} C_{1}^{2}-48 \Omega_{2} C_{1}}
$$

with $Z_{2}=N_{2}^{2}$.

It is noticeable that equation (44) is similar with a commonly encountered differential equation that describes the transient evolution of targeted energy transfer within a single degree-of-freedom system attached to a NES. Multiple bifurcated solutions are obtained by seeking the roots of related polynomial mapping:

$$
P\left(Z_{2}\right)=27 \Omega_{2}^{2} Z_{2}^{2}-\left(48 \Omega_{2}-72 \Omega_{2}^{2} C_{1}\right) Z_{2}+16 \gamma_{2}^{2}+16+36 \Omega_{2}^{2} C_{1}^{2}-48 \Omega_{2} C_{1}
$$

Targeted energy transfer $\mathrm{TET}_{2}$ in $\psi_{4}$ may be witnessed when energy drops down from one solution to another more stable one which means that $P\left(Z_{2}\right)$ must have at least two positive roots. This leads to the following conditions:

$$
\left\{\begin{array}{cc}
C_{1} & <\frac{2}{3 \Omega_{2}} \\
\left(1-\frac{3}{2} \Omega_{2} C_{1}+\sqrt{3} \gamma_{2}\right)\left(1-\frac{3}{2} \Omega_{2} C_{1}-\sqrt{3} \gamma_{2}\right) & >0
\end{array}\right.
$$

\footnotetext{
${ }^{2}$ In fact, $C_{1}$ may indeed be understood as a time delayed initial energy for $\psi_{2}=\lim _{T_{0} \rightarrow \infty} \varphi_{2}$ which practically means after a certain amount of time $T_{0}$ has lasted.
} 
or similarly

$$
C_{1}<S_{1} \quad \text { with } S_{1}=\frac{2}{3} \frac{1-\sqrt{3} \frac{\lambda}{\omega_{2}}}{K} \omega_{2}^{2}
$$

involving an activation energy threshold $S_{1}$.

As a consequence, targeted energy transfer $\mathrm{TET}_{2}$ is triggered towards the second differential mode $\psi_{4}$ when condition (47) is satisfied. By considering the limit $\tau_{1} \rightarrow \infty$, one trivially obtains that $N_{2} \rightarrow 0$ and equations (39) finally reduces to:

$$
\begin{cases}\frac{1}{2} \lambda \omega_{1} \psi_{2}-\frac{3}{8} i K \psi_{2}\left|\psi_{2}\right|^{2}-\frac{1}{2} i \omega_{1}^{2} \varphi_{1}+\frac{1}{2} i \omega_{1}^{2} \psi_{2} & =0 \\ \frac{\partial \varphi_{1}}{\partial T_{1}}+\frac{1}{2} i \omega_{1} \varphi_{1}-\frac{1}{2} i \omega_{1} \psi_{2} & =0\end{cases}
$$

with $\Omega_{1}=\frac{K}{\omega_{1}^{2}}$ and $\gamma_{1}=\frac{\lambda}{\omega_{1}}$.

Problem (39) is hence transformed into a classical targeted energy transfer problem between a single master degree-of-freedom and a NES. A simple explanation may here be given to improve the physical understanding of what is going on. Targeted energy transfer mechanism in $\psi_{4}$ is winning against its competitor in $\psi_{2}$ when characteristic times of both mechanisms are well separated i.e. nonlinear coupling in $\psi_{1}$ equations is much greater that its counterpart in $\psi_{3}$ equations. As a result, a relatively large coupling in $\psi_{1}$ equations prevents to feed energy to its related localized mode.

Generally speaking, ratio $\frac{\epsilon_{1}}{\epsilon_{2}}$ is strongly depending on generalized modal masses and mode shape amplification factors at the attachment. Although very helpful to understand TET mechanisms, case study $\epsilon_{1} \ll \epsilon_{2}$ happens to be a little bit restrictive. Next sections also aim to investigate competitive energy transfers when small parameters $\epsilon_{1}$ and $\epsilon_{2}$ have the same order of magnitude and to provide TET scenarios likely to occur.

\subsection{Basic mechanism for competitive TETs}

Coming back to the general case with no assumption made upon natural frequencies $\omega_{1}$ and $\omega_{2}$, one is now interested in how targeted energy transfer $\mathrm{TET}_{1}$ in $\psi_{2}$ is competing against targeted energy transfer $\mathrm{TET}_{2}$ in $\psi_{4}$ with respect to time. The basic idea is to consider that $\mathrm{TET}_{1}$ and $\mathrm{TET}_{2}$ events are triggered within almost instantaneous laps of time and that it is reasonable to claim that for instance $\left|\psi_{4}\right|$ remains piecewise constant time-dependent while $\mathrm{TET}_{1}$ is active until a given activation energy threshold is went through.

Whatever natural frequencies $\omega_{1}$ and $\omega_{2}$, one may always assume that time scale $\omega_{1} T_{1}=\epsilon_{1} \omega_{1} t$ for $\operatorname{TET}_{1}$ in $\psi_{2}$ is different from time scale $\omega_{2} \tau_{1}=\epsilon_{2} \omega_{2} t$ according to $\mathrm{TET}_{2}$ in $\psi_{4}$. Hence, it is possible to follow the simultaneous response of two quantities when time scales $\omega_{1} T_{1}$ and $\omega_{2} \tau_{1}$ become neighbors. 
By assuming that

$$
C_{2}=\left|\psi_{4}\right|^{2}
$$

is a piecewise constant with respect to time, one may rewrite system of equations (39) like:

$$
\begin{cases}\frac{1}{2} \lambda \omega_{1} \psi_{2}-\frac{3}{8} i K \psi_{2}\left|\psi_{2}\right|^{2}-\frac{1}{2} i \omega_{1}^{2} \varphi_{1}+\frac{1}{2} i \omega_{1}^{2} \psi_{2}-\frac{3}{4} i K C_{2} \psi_{2} & =0 \\ \frac{\partial \varphi_{1}}{\partial T_{1}}+\frac{1}{2} i \omega_{1} \varphi_{1}-\frac{1}{2} i \omega_{1} \psi_{2} & =0\end{cases}
$$

and $Z_{1}=N_{1}^{2}$ appears to be a solution of equation:

$$
\frac{d Z_{1}}{d T_{1}}=-\frac{16 \omega_{1} \gamma_{1} Z_{1}}{27 \Omega_{1}^{2} Z_{1}^{2}-\left(48 \Omega_{1}-72 \Omega_{1}^{2} C_{2}\right) Z_{1}+16 \gamma_{1}^{2}+16+36 \Omega_{1}^{2} C_{1}^{2}-48 \Omega_{1} C_{2}}
$$

Similarly to equation (47), one may exhibit another activation energy threshold $S_{2}$ defined by

$$
C_{2}<S_{2} \quad \text { with } S_{2}=\frac{2}{3} \frac{1-\sqrt{3} \frac{\lambda}{\omega_{1}}}{K} \omega_{1}^{2}
$$

Activation energy thresholds $S_{1}$ and $S_{2}$ that constitute upper bounds for targeted energy transfers and are invariant upon different choices of essential nonlinear stiffness or damping of NES, are prerequisite for triggering targeted energy transfers $\mathrm{TET}_{2}$ and $\mathrm{TET}_{1}$ respectively. By contrast, lower bound for energy thresholds indeed exist and they are time varying quantities likely to diminish mainly due to the occurrence of dissipation in related NNMs.

Several scenarios for targeted energy transfers detailed hereafter may also be derived qualitatively by exploring whether conditions (47) and (52) are satisfied or not.

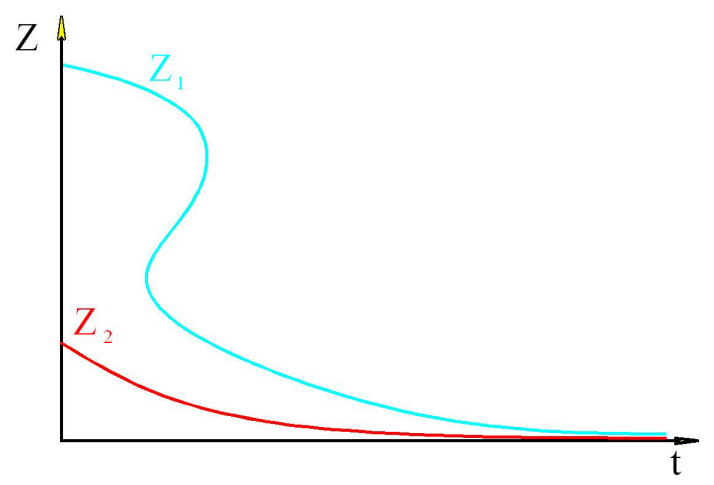

Figure 2: Scenario $C_{1} \geq S_{1} ; C_{2} \leq S_{2}$ : TET 1 mechanism is solely active

\subsection{Scenario $C_{1} \geq S_{1} \Rightarrow \mathrm{TET}_{2}$ disabled ; $C_{2} \leq S_{2} \Rightarrow \mathrm{TET}_{1}$ enabled}

Here initial energy is assumed to be mainly concentrated upon the first internal mode with checked condition $C_{1} \geq S_{1}$ which prevents $\mathrm{TET}_{2}$ mechanism to settle down. Condition $C_{2} \leq S_{2}$ is moreover 
assumed which means that $\mathrm{TET}_{1}$ is free to be triggered. In present configuration that is the most commonly encountered case, energy is not high enough to trigger a bifurcation jump in second internal mode and this latter is attenuated by its natural dissipation versus time. At the same time, the existence of multiple solutions for the first internal mode is ensured by condition $C_{2} \leq S_{2}$ and this latter undergoes a bifurcation jump from the higher energy solution towards the more stable solution, thus triggering $\mathrm{TET}_{1}$ mechanism. The time response of energy embedded in internal mode 1 merely depends on initial energy sprayed to internal mode 2 and on the choice of both nonlinear stiffness and damping of the NES attachment. As a result, dynamic equations may be uncoupled for $C_{2}$ plays a minor role in systems of equations (39) and (40). This scenario also brings back to the well known study of a single degree-of-freedom master system attached to a NES.

In that case, the trend of energy decay in internal mode 1 is given by:

$$
\frac{d Z_{1}}{d T_{1}} \approx-\frac{16 \omega_{1} \gamma_{1} Z_{1}}{27 \Omega_{1}^{2} Z_{1}^{2}-\left(48 \Omega_{1}-72 \Omega_{1}^{2} C_{2}\right) Z_{1}+16 \gamma_{1}^{2}+16+36 \Omega_{1}^{2} C_{1}^{2}}
$$

or similarly by implicit formulae

$$
\frac{27}{2} \Omega_{1}^{2}\left(Z_{1}^{2}-Z_{1_{0}}^{2}\right)-48 \Omega_{1}\left(Z_{1}-Z_{1_{0}}\right)+\left(16\left(1+\gamma_{1}^{2}\right)+36 \Omega_{1}^{2} C_{1}^{2}\right) \ln \left|\frac{Z_{1}}{Z_{1_{0}}}\right| \approx-16 \omega_{1} \gamma_{1} T_{1}
$$

whereas energy decay in internal mode 2 is depicted by:

$$
\frac{d Z_{2}}{d \tau_{1}} \approx-\frac{\lambda}{1+\frac{\lambda^{2}}{\omega_{2}^{2}}} Z_{2}
$$

or similarly by:

$$
Z_{2} \approx Z_{2_{0}} \exp \left(\frac{\lambda \tau_{1}}{1+\frac{\lambda^{2}}{\omega_{2}^{2}}}\right)
$$

From a qualitative point of view, transient trend of energy decay $Z_{1}$ and $Z_{2}$ for internal modes 1 and 2 respectively is displayed in Fig. 2.

\subsection{Scenario $C_{1} \geq S_{1} ; C_{2} \geq S_{2} \Rightarrow \mathrm{TET}_{1}$ and $\mathrm{TET}_{2}$ in wait for being triggered}

Here, initial energy $C_{1}$ and $C_{2}$ of internal modes 1 and 2 are assumed to be greater than their corresponding activation threshold $S_{1}$ and $S_{2}$. Present scenario sketches what is very likely to occur in the triggering of $\mathrm{TET}_{1}$ and $\mathrm{TET}_{2}$ mechanisms:

step 1 : At initial time, no TET mechanism is developing in the studied system. Due to the occurrence of dissipation in both internal modes 1 and 2 , energies $Z_{1}$ (resp. $Z_{2}$ ) decay until one is going through its related activation threshold $S_{2}$ (resp. $S_{1}$ ),

step 2 : One energy threshold $S_{1}$ or $S_{2}$ is reached. By considering that initial energy is equally spread onto internal modes 1 and 2 and assuming that modal frequencies satisfy relationship $\omega_{1}<\omega_{2}$ which 
results in $S_{2}<S_{1}$, energy $Z_{1}$ is likely to go down its activation energy boundary $S_{1}$ first while at the same time $Z_{2}$ remains above $S_{2}$. Hence, there is a possibility for $\mathrm{TET}_{2}$ mechanism to settle down. From this point, curve shape of $Z_{2}$ is virtually modified for future evolution and a new condition upon energy is required to trigger $\mathrm{TET}_{2}$,

step 3 : Energy $Z_{1}$ goes on decaying due to damping and curve shape of $Z_{2}$ is modified accordingly through nonlinear coupling components. A virtual bifurcation jump for future evolution becomes steeper and the probability of $\mathrm{TET}_{2}$ to occur is raised,

step 4 : Energy $Z_{2}$ is high enough to trigger targeted energy transfer $\mathrm{TET}_{2}$ involving internal mode 2 and $Z_{2}$ instantaneously drops down to a more stable energy level with no link with $C_{1}$. Energy $Z_{2}$ fastly decays until condition $Z_{2} \leq S_{2}$ is encountered which again gives a chance to TET 1 to eventually occur,

step 5 : If energy $Z_{1}$ is sufficient, TET $_{1}$ mechanism is also triggered onto internal mode 1 , and solution $Z_{1}$ jumps down instantaneously to a more stable energy level,

step 6 : Neither conditions $Z_{1} \geq S_{1}$ nor $Z_{2} \geq S_{2}$ are satisfied. Both $\mathrm{TET}_{1}$ and $\mathrm{TET}_{2}$ mechanisms are disabled. Energies $Z_{1}$ and $Z_{2}$ continue to decay due to natural damping.

Main mechanisms involved in this scenario are step by step illustrated in Fig. 3. Because the decaying trend of energy $Z_{2}$ is closely related to the one of $Z_{1}$, equations (39) and (40) may not be easily uncoupled. Next section aims also introduces a degenerate case that enables to partly uncouple equations of trends.

\subsection{Degenerate equations of motion using a single time scale}

Previous analytical developments introduced independent time scales $\left(T_{i}\right)_{i}$ and $\left(\tau_{i}\right)_{i}$ involving small non co-mensurable parameters $\epsilon_{1}$ and $\epsilon_{2}$ to derive equations of motion (39) and (40) in $\varphi_{1}, \varphi_{3}, \psi_{2}$ and $\psi_{4}$. Yet, it is reasonable to claim that time scales $T_{1}$ and $\tau_{1}$ are very close to each other and that they could be considered as dependant time variables constrained by for instance the following relationship:

$$
\alpha=\frac{\tau_{1}}{T_{1}}=\frac{\epsilon_{2}}{\epsilon_{1}}
$$

The damping related to the primary mass $\lambda_{1}$ and $\lambda_{2}$ can be taken into account here by ignoring condition (26), equations (39) and (40) hence become:

$$
\begin{cases}\frac{1}{2} \lambda \omega_{1} \psi_{2}-\frac{3}{8} i K \psi_{2}\left|\psi_{2}\right|^{2}-\frac{1}{2} i \omega_{1}^{2} \varphi_{1}+\frac{1}{2} i \omega_{1}^{2} \psi_{2}-\frac{3}{4} i K \psi_{2}\left|\psi_{4}\right|^{2} & =0 \\ \frac{\partial \varphi_{1}}{\partial T_{1}}+\frac{1}{2} \lambda_{1} \varphi_{1}+\frac{1}{2} i \omega_{1} \varphi_{1}-\frac{1}{2} i \omega_{1} \psi_{2} & =\end{cases}
$$

and

$$
\begin{cases}\frac{1}{2} \lambda \omega_{2} \psi_{4}-\frac{3}{8} i K \psi_{4}\left|\psi_{4}\right|^{2}-\frac{1}{2} i \omega_{2}^{2} \varphi_{3}+\frac{1}{2} i \omega_{2}^{2} \psi_{4}-\frac{3}{4} i K \psi_{4}\left|\psi_{2}\right|^{2} & =0 \\ \frac{1}{\alpha} \frac{\partial \varphi_{3}}{\partial T_{1}}+\frac{1}{2} \lambda_{2} \varphi_{3}+\frac{1}{2} i \omega_{2} \varphi_{3}-\frac{1}{2} i \omega_{2} \psi_{4} & =0\end{cases}
$$



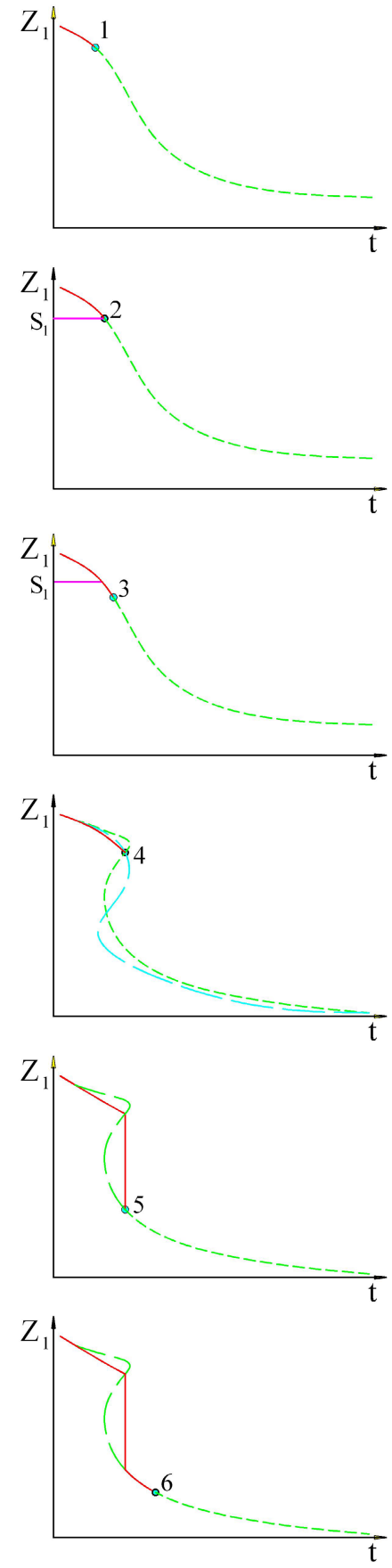

step 1

step 2

step 3

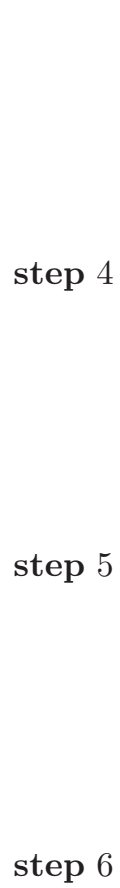

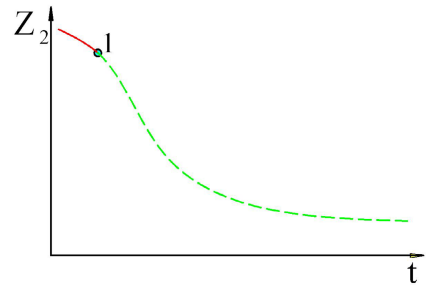
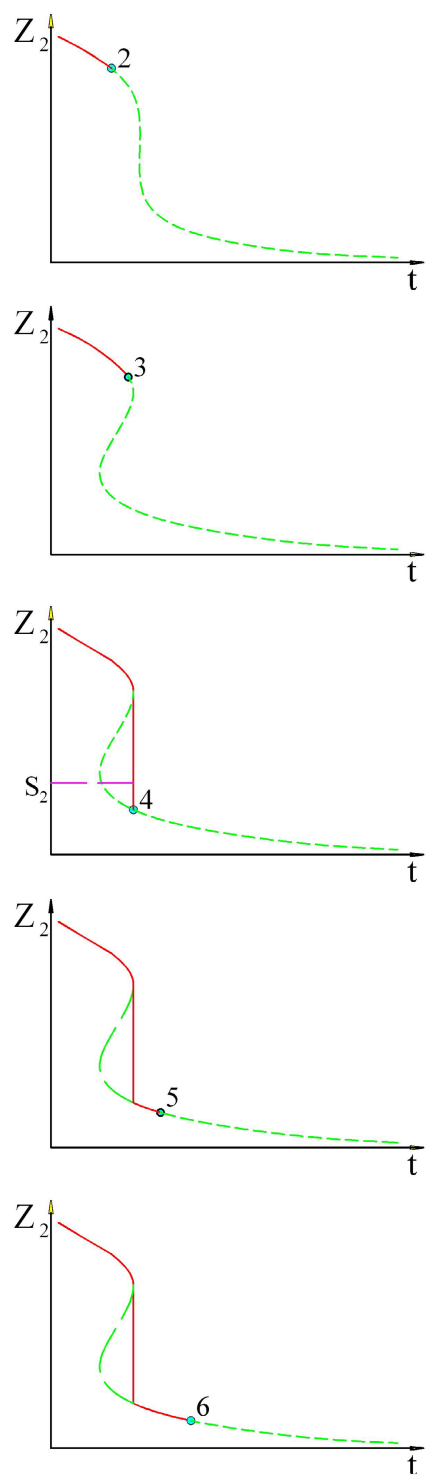

Figure 3: Scenario $C_{1} \geq S_{1} ; C_{2} \geq S_{2}$ : $\mathrm{TET}_{1}$ and $\mathrm{TET}_{2}$ mechanisms eventually active in sequence ; steps 1 to 6 .

featuring functions $\varphi_{3}$ and $\psi_{4}$ that now solely depend on time $T_{1}$. 
By expanding equations (39) and (59) using the following polar coordinates:

$$
\begin{aligned}
& \psi_{2}=N_{1} e^{i \delta_{1}} \\
& \psi_{4}=N_{2} e^{i \delta_{2}}
\end{aligned}
$$

new equations of motion in energy amplitudes $N_{1}$ and $N_{2}$ may be obtained as:

$$
\left\{\begin{array}{l}
\frac{d N_{1}}{d T_{1}}=\frac{P_{1}\left(N_{1}, N_{2}\right)}{P\left(N_{1}, N_{2}\right)} \\
\frac{d N_{2}}{d T_{1}}=\frac{P_{2}\left(N_{1}, N_{2}\right)}{P\left(N_{1}, N_{2}\right)}
\end{array}\right.
$$

with

$$
P_{1}\left(N_{1}, N_{2}\right)=-324 \Omega_{1}^{2} \Omega_{2}^{2} \lambda_{1} N_{1}^{9}+\left(432 \Omega_{1}^{2} \Omega_{2} \lambda_{1}+864 \lambda_{1} \Omega_{2}^{2} \Omega_{1}\right) N_{1}^{7}+\left(1296 \Omega_{1}^{2} \Omega_{2}^{2} \lambda_{2}-1944 \Omega_{1}^{2} \Omega_{2}^{2} \lambda_{1}\right) N_{2}^{2} N_{1}^{7}-
$$
$\left(144 \Omega_{1}^{2} \gamma_{2}^{2} \lambda_{1}+1152 \lambda_{1} \Omega_{1} \Omega_{2}+576 \lambda_{1} \gamma_{1}^{2} \Omega_{2}^{2}+144 \Omega_{1}^{2} \lambda_{1}+576 \Omega_{2}^{2} \omega_{1} \gamma_{1}+576 \lambda_{1} \Omega_{2}^{2}\right) N_{1}^{5}+\Omega_{1}^{2} \Omega_{2}^{2}\left(3888 \lambda_{2}-4131 \lambda_{1}\right) N_{2}^{4} N_{1}^{5}+$ $\left(3456 \lambda_{1} \Omega_{2}^{2} \Omega_{1}+2160 \Omega_{1}^{2} \Omega_{2} \lambda_{1}-1728 \Omega_{1}^{2} \lambda_{2} \Omega_{2}-1728 \Omega_{1} \Omega_{2}^{2} \lambda_{2}\right) N_{2}^{2} N_{1}^{5}+\left(768 \Omega_{2} \omega_{1} \gamma_{1}+768 \lambda_{1} \gamma_{1}^{2} \Omega_{2}+384 \lambda_{1} \Omega_{1}+\right.$ $\left.384 \gamma_{2}^{2} \lambda_{1} \Omega_{1}+768 \lambda_{1} \Omega_{2}\right) N_{1}^{3}+\left(3456 \Omega_{1}^{2} \Omega_{2} \lambda_{1}+4104 \lambda_{1} \Omega_{2}^{2} \Omega_{1}-1728 \Omega_{1} \Omega_{2}^{2} \lambda_{2}-4320 \Omega_{1}^{2} \lambda_{2} \Omega_{2}\right) N_{2}^{4} N_{1}^{3}+\left(2916 \Omega_{1}^{2} \Omega_{2}^{2} \lambda_{2}-\right.$ $\left.3564 \Omega_{1}^{2} \Omega_{2}^{2} \lambda_{1}\right) N_{2}^{6} N_{1}^{3}+\left(-576 \Omega_{1}^{2} \gamma_{2}^{2} \lambda_{1}-1152 \lambda_{1} \Omega_{2}^{2}-3456 \lambda_{1} \Omega_{1} \Omega_{2}-1152 \Omega_{2}^{2} \omega_{1} \gamma_{1}-576 \Omega_{1}^{2} \lambda_{1}+576 \lambda_{2} \gamma_{2}^{2} \Omega_{1}^{2}+\right.$ $\left.2304 \Omega_{1} \lambda_{2} \Omega_{2}+576 \Omega_{1}^{2} \alpha \omega_{2} \gamma_{2}+576 \Omega_{1}^{2} \lambda_{2}-1152 \lambda_{1} \gamma_{1}^{2} \Omega_{2}^{2}\right) N_{2}^{2} N_{1}^{3}+\left(-256 \lambda_{1}-256 \omega_{1} \gamma_{1}-256 \lambda_{1} \gamma_{1}^{2}-256 \lambda_{1} \gamma_{1}^{2} \gamma_{2}^{2}-\right.$ $\left.256 \lambda_{1} \gamma_{2}^{2}-256 \gamma_{2}^{2} \omega_{1} \gamma_{1}\right) N_{1}+\left(-432 \lambda_{1} \gamma_{1}^{2} \Omega_{2}^{2}+1152 \Omega_{1}^{2} \alpha \omega_{2} \gamma_{2}-432 \Omega_{2}^{2} \omega_{1} \gamma_{1}+1152 \Omega_{1} \lambda_{2} \Omega_{2}-2304 \lambda_{1} \Omega_{1} \Omega_{2}-\right.$ $\left.576 \Omega_{1}^{2} \lambda_{1}+1152 \lambda_{2} \gamma_{2}^{2} \Omega_{1}^{2}-576 \Omega_{1}^{2} \gamma_{2}^{2} \lambda_{1}+1152 \Omega_{1}^{2} \lambda_{2}-432 \lambda_{1} \Omega_{2}^{2}\right) N_{2}^{4} N_{1}+\left(-972 \Omega_{1}^{2} \Omega_{2}^{2} \lambda_{1}+648 \Omega_{1}^{2} \Omega_{2}^{2} \lambda_{2}\right) N_{2}^{8} N_{1}+$ $\left(-432 \Omega_{1} \Omega_{2}^{2} \lambda_{2}+1296 \lambda_{1} \Omega_{2}^{2} \Omega_{1}+1728 \Omega_{1}^{2} \Omega_{2} \lambda_{1}-1728 \Omega_{1}^{2} \lambda_{2} \Omega_{2}\right) N_{2}^{6} N_{1}+\left(768 \gamma_{2}^{2} \lambda_{1} \Omega_{1}-768 \Omega_{1} \alpha \omega_{2} \gamma_{2}-768 \lambda_{2} \gamma_{2}^{2} \Omega_{1}+\right.$ $\left.768 \lambda_{1} \gamma_{1}^{2} \Omega_{2}+768 \Omega_{2} \omega_{1} \gamma_{1}+768 \lambda_{1} \Omega_{1}-768 \Omega_{1} \lambda_{2}+768 \lambda_{1} \Omega_{2}\right) N_{2}^{2} N_{1}$

$P_{2}\left(N_{1}, N_{2}\right)=\left(-972 \Omega_{1}^{2} \Omega_{2}^{2} \lambda_{2}+648 \Omega_{1}^{2} \Omega_{2}^{2} \lambda_{1}\right) N_{2} N_{1}^{8}+\left(2916 \Omega_{1}^{2} \Omega_{2}^{2} \lambda_{1}-3564 \Omega_{1}^{2} \Omega_{2}^{2} \lambda_{2}\right) N_{1}^{6} N_{2}^{3}+\left(1728 \Omega_{1} \Omega_{2}^{2} \lambda_{2}-\right.$ $\left.1728 \lambda_{1} \Omega_{2}^{2} \Omega_{1}+1296 \Omega_{1}^{2} \lambda_{2} \Omega_{2}-432 \Omega_{1}^{2} \Omega_{2} \lambda_{1}\right) N_{1}^{6} N_{2}+\left(3888 \Omega_{1}^{2} \Omega_{2}^{2} \lambda_{1}-4131 \Omega_{1}^{2} \Omega_{2}^{2} \lambda_{2}\right) N_{1}^{4} N_{2}^{5}+\left(-1728 \Omega_{1}^{2} \Omega_{2} \lambda_{1}-\right.$ $\left.4320 \lambda_{1} \Omega_{2}^{2} \Omega_{1}+4104 \Omega_{1}^{2} \lambda_{2} \Omega_{2}+3456 \Omega_{1} \Omega_{2}^{2} \lambda_{2}\right) N_{1}^{4} N_{2}^{3}+\left(1152 \Omega_{2}^{2} \omega_{1} \gamma_{1}-2304 \Omega_{1} \lambda_{2} \Omega_{2}-576 \lambda_{2} \Omega_{2}^{2}-432 \lambda_{2} \gamma_{2}^{2} \Omega_{1}^{2}-\right.$ $\left.432 \Omega_{1}^{2} \lambda_{2}+1152 \lambda_{1} \Omega_{2}^{2}-432 \Omega_{1}^{2} \alpha \omega_{2} \gamma_{2}+1152 \lambda_{1} \Omega_{1} \Omega_{2}-576 \lambda_{2} \Omega_{2}^{2} \gamma_{1}^{2}+1152 \lambda_{1} \gamma_{1}^{2} \Omega_{2}^{2}\right) N_{1}^{4} N_{2}+\left(-1944 \Omega_{1}^{2} \Omega_{2}^{2} \lambda_{2}+\right.$ $\left.1296 \Omega_{1}^{2} \Omega_{2}^{2} \lambda_{1}\right) N_{1}^{2} N_{2}^{7}+\left(2160 \Omega_{1} \Omega_{2}^{2} \lambda_{2}+3456 \Omega_{1}^{2} \lambda_{2} \Omega_{2}-1728 \Omega_{1}^{2} \Omega_{2} \lambda_{1}-1728 \lambda_{1} \Omega_{2}^{2} \Omega_{1}\right) N_{1}^{2} N_{2}^{5}+\left(-3456 \Omega_{1} \lambda_{2} \Omega_{2}-\right.$ $1152 \lambda_{2} \gamma_{2}^{2} \Omega_{1}^{2}+576 \lambda_{1} \Omega_{2}^{2}-576 \lambda_{2} \Omega_{2}^{2}-576 \lambda_{2} \Omega_{2}^{2} \gamma_{1}^{2}-1152 \Omega_{1}^{2} \alpha \omega_{2} \gamma_{2}+576 \Omega_{2}^{2} \omega_{1} \gamma_{1}-1152 \Omega_{1}^{2} \lambda_{2}+576 \lambda_{1} \gamma_{1}^{2} \Omega_{2}^{2}+$ $\left.2304 \lambda_{1} \Omega_{1} \Omega_{2}\right) N_{1}^{2} N_{2}^{3}+\left(768 \Omega_{1} \lambda_{2}-768 \lambda_{1} \Omega_{2}+768 \lambda_{2} \gamma_{2}^{2} \Omega_{1}-768 \lambda_{1} \gamma_{1}^{2} \Omega_{2}-768 \Omega_{2} \omega_{1} \gamma_{1}+768 \lambda_{2} \Omega_{2}+768 \Omega_{1} \alpha \omega_{2} \gamma_{2}+\right.$ $\left.768 \lambda_{2} \Omega_{2} \gamma_{1}^{2}\right) N_{1}^{2} N_{2}-324 \Omega_{1}^{2} \Omega_{2}^{2} \lambda_{2} N_{2}^{9}+\left(432 \Omega_{1} \Omega_{2}^{2} \lambda_{2}+864 \Omega_{1}^{2} \lambda_{2} \Omega_{2}\right) N_{2}^{7}+\left(-576 \Omega_{1}^{2} \lambda_{2}-144 \lambda_{2} \Omega_{2}^{2}-576 \Omega_{1}^{2} \alpha \omega_{2} \gamma_{2}-\right.$ $\left.144 \lambda_{2} \Omega_{2}^{2} \gamma_{1}^{2}-576 \lambda_{2} \gamma_{2}^{2} \Omega_{1}^{2}-1152 \Omega_{1} \lambda_{2} \Omega_{2}\right) N_{2}^{5}+\left(768 \Omega_{1} \lambda_{2}+384 \lambda_{2} \Omega_{2} \gamma_{1}^{2}+384 \lambda_{2} \Omega_{2}+768 \lambda_{2} \gamma_{2}^{2} \Omega_{1}+768 \Omega_{1} \alpha \omega_{2} \gamma_{2}\right) N_{2}^{3}+$ $\left(-256 \lambda_{2}-256 \lambda_{2} \gamma_{2}^{2}-256 \alpha \omega_{2} \gamma_{2}-256 \lambda_{2} \gamma_{2}^{2} \gamma_{1}^{2}-256 \lambda_{2} \gamma_{1}^{2}-256 \gamma_{1}^{2} \alpha \omega_{2} \gamma_{2}\right) N_{2}$,

$$
P\left(N_{1}, N_{2}\right)=512+512 \gamma_{2}^{2} \gamma_{1}^{2}+512 \gamma_{1}^{2}+512 \gamma_{2}^{2}+1458 \Omega_{1}^{2} N_{2}^{4} \Omega_{2}^{2} N_{1}^{4}+1944 \Omega_{2}^{2} N_{1}^{8} \Omega_{1}^{2}+\left(4608 \Omega_{2} \Omega_{1}+864 \Omega_{2}^{2} \gamma_{1}^{2}+\right.
$$
$\left.864 \Omega_{2}^{2}+1152 \Omega_{1}^{2}+1152 \gamma_{2}^{2} \Omega_{1}^{2}\right) N_{2}^{4}+\left(-2592 \Omega_{2} \Omega_{1}^{2}-3456 \Omega_{2}^{2} \Omega_{1}\right) N_{1}^{6}+1944 \Omega_{2}^{2} N_{2}^{8} \Omega_{1}^{2}+\left(-1536 \Omega_{2}-1536 \Omega_{1}-\right.$ $\left.1536 \gamma_{2}^{2} \Omega_{1}-1536 \Omega_{2} \gamma_{1}^{2}\right) N_{1}^{2}+\left(-3456 \Omega_{2} \Omega_{1}^{2}-2592 \Omega_{2}^{2} \Omega_{1}\right) N_{2}^{6}+\left(-1536 \Omega_{2}-1536 \Omega_{1}-1536 \gamma_{2}^{2} \Omega_{1}-1536 \Omega_{2} \gamma_{1}^{2}\right) N_{2}^{2}+$ $\left(864 \Omega_{1}^{2}+4608 \Omega_{2} \Omega_{1}+864 \gamma_{2}^{2} \Omega_{1}^{2}+1152 \Omega_{2}^{2}+1152 \Omega_{2}^{2} \gamma_{1}^{2}\right) N_{1}^{4}+\left(2304 \gamma_{2}^{2} \Omega_{1}^{2}+4608 \Omega_{2} \Omega_{1}+2304 \Omega_{2}^{2} \gamma_{1}^{2}+2304 \Omega_{2}^{2}+\right.$ $\left.2304 \Omega_{1}^{2}\right) N_{1}^{2} N_{2}^{2}+3888 \Omega_{1}^{2} N_{2}^{6} \Omega_{2}^{2} N_{1}^{2}+3888 \Omega_{1}^{2} N_{2}^{2} \Omega_{2}^{2} N_{1}^{6}+\left(-3456 \Omega_{2} \Omega_{1}^{2}-6048 \Omega_{2}^{2} \Omega_{1}\right) N_{2}^{4} N_{1}^{2}+\left(-3456 \Omega_{2}^{2} \Omega_{1}-\right.$ 
$\left.6048 \Omega_{2} \Omega_{1}^{2}\right) N_{2}^{2} N_{1}^{4}$

Equations of motion (39) and (40) finally reduce to a set of partly uncoupled equations (62) that may easily be integrated to exhibit the dynamic behavior of $N_{1}$ and $N_{2}$ with respect to time scale $T_{1}$. Eventual bifurcation jumps ie triggering of $\mathrm{TET}_{1}$ and $\mathrm{TET}_{2}$ mechanisms are here synchronous and related to the roots of polynomial $P\left(N_{1}, N_{2}\right)$ involved in equations (62). Next section introduces an academic numerical instance to highlight the relevance of $\mathrm{TET}_{s}$ scenarios discussed here above.

\section{Numerical experiments}

A benchmark application featuring a two bay frame civil engineering structure is used to assess the reliability of proposed targeted energy transfers mechanisms. Here the two degrees-of-freedom building model is connected to essentially nonlinear absorber that can resonate with either the first or the second eigenmode of the master structure. Two targeted energy transfers are also in competition for mitigating vibration induced in the primary structure. Numerical simulations achieved in the case of a free oscillation regime or with a pulse-like excitation enable to assess the validity of proposed scenarios for energy pumping by comparing energy trends obtained by integrating both degenerate analytical equations of motion and original model.
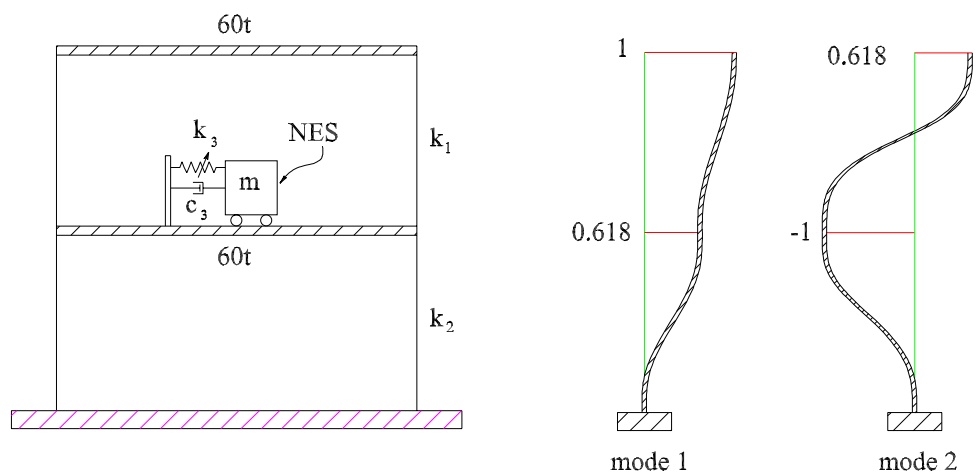

Figure 4: left: A two storey building model coupled with a NES ; center: modal deflection $\phi_{1}$; right: modal deflection $\phi_{2}$.

\subsection{A two storey building model}

A reduced order model of an actual two storey building, presented in [30], is here considered as a benchmark application. The building is made of two concrete slabs weighting 60 tons each as depicted in Fig. 4. Slabs are connected to a set of vertical steel beams themselves clamped on a concrete fondation. A purely nonlinear absorber (NES) is additionally mounted on first floor slab. 


\section{Modal properties}

A discrete skewer model shown in Fig. 5 is obtained by considering concentrated masses at the center of slabs and equivalent bending stiffness given by:

$$
k_{1}=k_{2}=\frac{24 E I}{h^{3}}=18.6 \times 10^{3} \mathrm{kN} / \mathrm{m}
$$

A standard modal analysis exhibits eigen-modes $\phi_{1}$ and $\phi_{2}$ of master system associated to natural frequencies:

- $f_{1}=1.73 \mathrm{~Hz}$,

- $f_{2}=4.53 \mathrm{~Hz}$.

whose unit-normalized modes shapes are illustrated in Fig. 4.

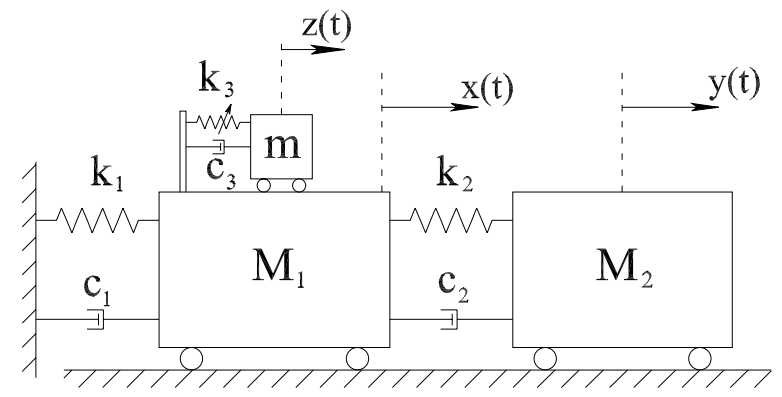

Figure 5: Discrete skewer model.

Mode shapes may firstly be written under matrix form:

$$
\underline{\underline{\Phi}}=\left[\begin{array}{ll}
\underline{\phi}_{1} & \underline{\phi}_{2}
\end{array}\right]=\left[\begin{array}{cc}
1 & -0.618 \\
0.618 & 1
\end{array}\right]
$$

and secondly mass-normalized according to the following rule:

- $M_{11}=M_{1} \phi_{11}^{2}+M_{2} \phi_{12}^{2}=82.91$ tons,

- $M_{22}=M_{1} \phi_{21}^{2}+M_{2} \phi_{22}^{2}=82.91$ tons.

Viscous damping matrix is assumed to be diagonal in the modal basis by using for instance Basile's rule with diagonal components chosen as in the realistic case:

- $c_{11}=3.61 \times 10^{3}$ N.m ${ }^{-1} . \mathrm{s}$,

- $c_{22}=23.6 \times 10^{3} \mathrm{~N} . \mathrm{m}^{-1} . \mathrm{s}$. 
Equations of motion are expanded onto the modal basis by using following change of coordinates

$$
[x, y]^{T}=\underline{\underline{\Phi}} \cdot\left[q_{1}, q_{2}\right]^{T}
$$

thus exhibiting modal coordinates $q_{1}$ and $q_{2}$ respectively for the first and the second mode; $y_{1}=\phi_{12} q_{1}$, $y_{2}=\phi_{22} q_{2}$.

All modal quantities such as mass, viscous damping and stiffness match variables introduced as in Fig. 1.

\section{NES design}

The nonlinear energy sink is designed by means of a small mass $m$ attached to mass $M_{1}$ with a pure cubic geometric nonlinearity in parallel with a linear viscous damper. A quick glance at Fig. 4 allows to notice that the NES is physically attached to a body of mode 2 and far away from the node of mode 1 which practically means that it is able to resonate with both modes. NES mass is chosen such that

$$
m=\epsilon_{1} \frac{M_{11}}{\phi_{12}^{2}}=2.7 \text { tons }
$$

corresponding to a mass ratio $\epsilon_{1}=\frac{1}{80}$ and $\epsilon_{2}=\frac{\phi_{22}^{2}}{\phi_{12}^{2}} \frac{M_{11}}{M_{22}} \epsilon_{1}=\frac{1}{31}$.

A typical value $k_{3}=4.05 \times 10^{6} \mathrm{kN} / \mathrm{m}^{3}$ that is not precisely matching an optimal design value is chosen for cubic nonlinear stiffness or similarly $K=0.15 \times 10^{3} \mathrm{kN} / \mathrm{m}^{3} / \mathrm{kg}$. Additionally a value $c_{3}=4.2 \times 10^{3} \mathrm{~N} \cdot \mathrm{m}^{-1}$.s is set arbitrarily for the NES or similarly $\lambda=1.55 \mathrm{~s}^{-1}$.

\section{TET energy thresholds}

Activation energy thresholds $S_{1}$ and $S_{2}$ may then be estimated from the knowledge of previous modal and NES parameters:

$$
\begin{aligned}
& S_{1}=\frac{2}{3} \frac{1-\sqrt{3} \frac{\lambda}{\omega_{2}}}{K} \omega_{2}^{2}=2.53 \times 10^{-4} \mathrm{~m}^{2} \\
& S_{2}=\frac{2}{3} \frac{1-\sqrt{3} \frac{\lambda}{\omega_{1}}}{K} \omega_{1}^{2}=1.15 \times 10^{-5} \mathrm{~m}^{2}
\end{aligned}
$$

\subsection{Validation of TET scenarios}

Two cases are considered hereafter to assess the relevance of competitive scenarios for targeted energy transfer as introduced in sections 3.3 and 3.4. Energies $\left(Z_{1}, Z_{2}\right)$ are numerically integrated using a Runge Kutta scheme $R K 45$ by using degenerated equations of motion (62).

scenario $\mathbf{C}_{\mathbf{1}} \leq \mathbf{S}_{\mathbf{1}} ; \mathbf{C}_{\mathbf{2}} \leq \mathbf{S}_{\mathbf{2}}$ : initial energies $C_{1}$ and $C_{2}$ are chosen such as: 


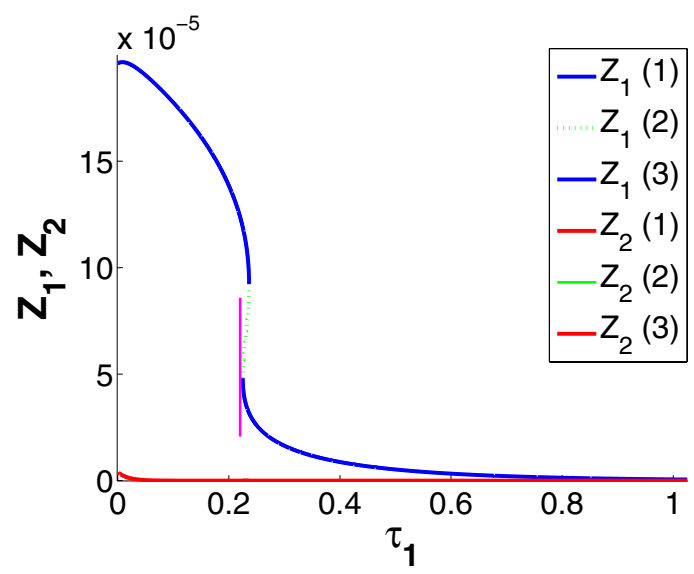

Figure 6: scenario $C_{1} \leq S_{1} ; C_{2} \leq S_{2}$.

- Initial energy for first internal mode: $C_{1}=2.25 \times 10^{-4} \mathrm{~m}^{2}$,

- Initial energy for second internal mode: $C_{2}=4 \times 10^{-6} \mathrm{~m}^{2}$.

In present configuration, $C_{2}$ is small enough so that $\mathrm{TET}_{2}$ mechanism is inactive ; on the first hand, second internal mode is attenuated by its natural damping and on the other hand, $\mathrm{TET}_{1}$ mechanism is enabled and a bifurcation jumped may be witnessed as depicted in Fig. 6.

scenario $\mathbf{C}_{\mathbf{1}} \leq \mathbf{S}_{\mathbf{1}} ; \mathbf{C}_{\mathbf{2}} \geq \mathbf{S}_{\mathbf{2}}$ : initial energies $\left|\varphi_{1}(0)\right|$ and $\left|\varphi_{3}(0)\right|$ are chosen such as:

- Initial energy for first internal mode:

$$
\begin{aligned}
\left|\varphi_{1}(0)\right| & \approx \sqrt{\left(\frac{\dot{y}_{1}(0)}{\omega_{1}}\right)^{2}+y_{1}(0)^{2}} \\
& \approx 0.01564,
\end{aligned}
$$

- Initial energy for second internal mode:

$$
\begin{aligned}
\left|\varphi_{3}(0)\right| & \approx \sqrt{\left(\frac{\dot{y}_{2}(0)}{\omega_{2}}\right)^{2}+y_{2}(0)^{2}} \\
& \approx 0.008432 .
\end{aligned}
$$

In order to validate previously mentioned analytical approach with numerical results, let's assume that the master system is without damping $\left(\lambda_{1}=0\right.$ and $\left.\lambda_{2}=0\right)$. First of all $\left|\psi_{2}(0)\right|$ and $\left|\psi_{4}(0)\right|$ should be evaluated by solving the first equations of system (39) and (40) with initial energies taken as follows:

$$
\left|\psi_{2}(0)\right|=0.0067122=N_{1}(0)=\sqrt{C_{1}}
$$

and

$$
\left|\psi_{4}(0)\right|=0.0122490=N_{2}(0)=\sqrt{C_{2}}
$$



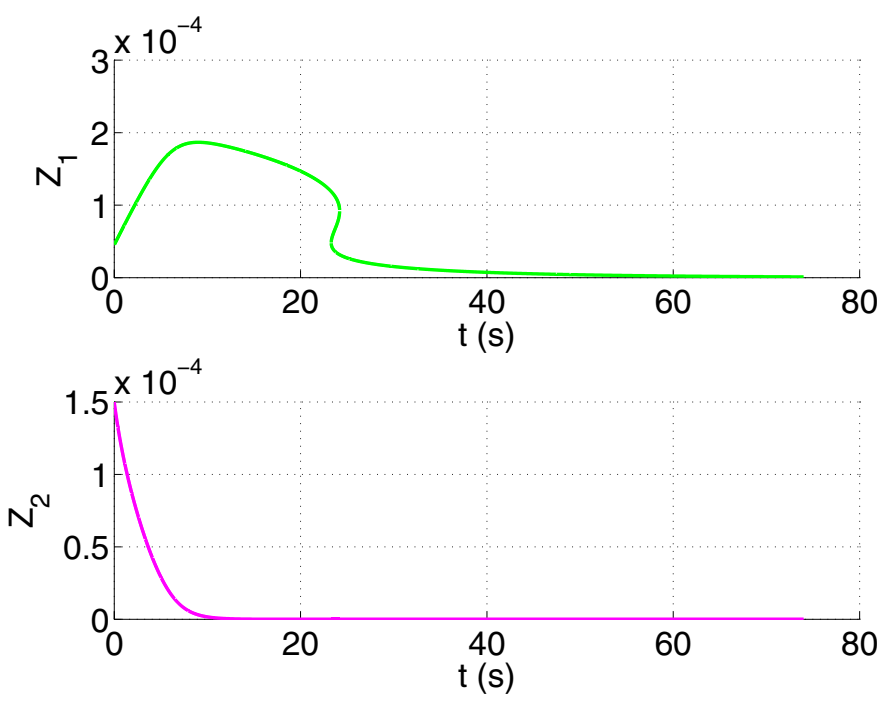

Figure 7: Evolution of the energy of relative modes.

In that case, it is noticeable in Fig. 7 that energy $Z_{2}$ decays fastly thanks to an active $\mathrm{TET}_{2}$ mechanism that is triggered instantaneously ${ }^{3}$. In the meantime, energy $Z_{1}$ is increasing ${ }^{4}$. As soon as condition $Z_{2} \leq S_{2}$ is satisfied, $\mathrm{TET}_{1}$ mechanism becomes active with a large energy jump.

The energy variation of the master system related to two modes are evaluated analytically and numerically and presented in Fig. 8. Results show an obvious agreement between numerical and analytical forecasts.

Energy trends $Z_{1}$ and $Z_{2}$ computed numerically are therefore in good agreement with forecasts provided by scenarios explained in sections 3.3 and 3.4 .

Results of energy transfer of the master system in the presence of the damping are introduced in Fig. 9.

\subsection{Dynamic behavior in free vibration regime}

Equations of motion (9) spanned onto the modal basis that involve quantities $\left(y_{1}, y_{2}, z\right)$ are numerically integrated in a free vibration regime to investigate the reliability of proposed scenarios and to check the efficiency of targeted energy transfers.

Initial velocities given by

- $y_{1}^{\prime}(0)=0.17 \mathrm{~m} / \mathrm{s}, y_{1}(0)=0.0 \mathrm{~m}$,

\footnotetext{
${ }^{3}$ It is important to stress the difficulty in determining initial energies for internal modes for initial conditions are not set at time scale $T_{1}=0$ but after some laps of time $T_{0}$ such that condition $T_{0} \rightarrow \infty$ becomes valid.

${ }^{4}$ Internal modes energies may increase at the beginning while energies related to the motion of the center of mass are decreasing.
} 

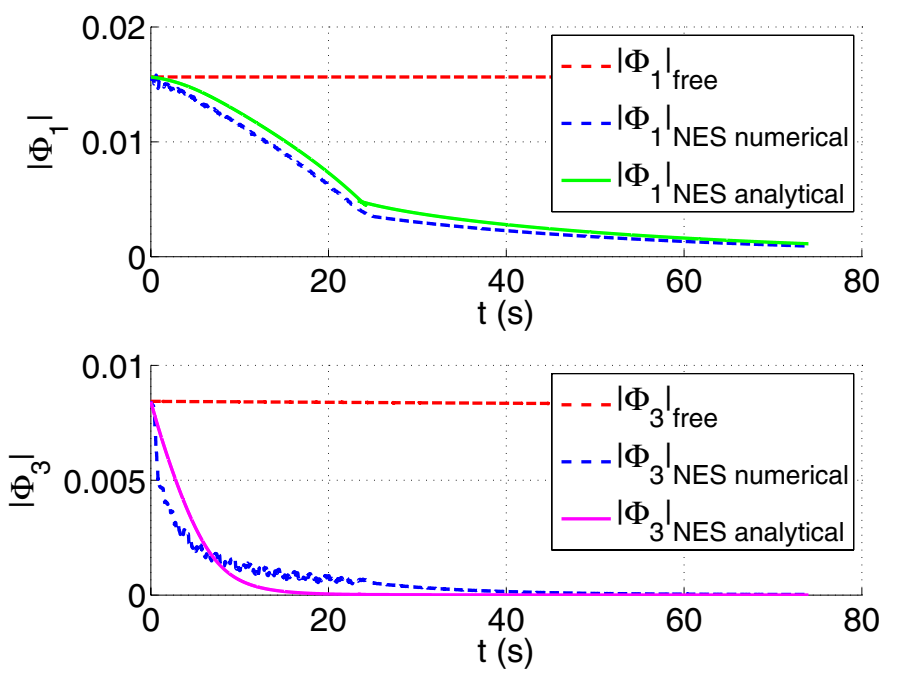

Figure 8: Comparison of energy transfer in the master system by using analytical and numerical methods

- $y_{2}^{\prime}(0)=0.27 \mathrm{~m} / \mathrm{s}, y_{2}(0)=0.0 \mathrm{~m}$,

- $z^{\prime}(0)=0.0 \mathrm{~m} / \mathrm{s}, z(0)=0.0 \mathrm{~m}$.

which yield maximum modal displacements

- $y_{1}(0)=1.5 \times 10^{-2} \mathrm{~m}$,

- $y_{2}(0)=0.5 \times 10^{-2} \mathrm{~m}$.

are chosen as initial conditions for the studied system.

A quick glance at Fig. 9.a) and 9.c) allows to claim that - apart a few peak oscillations remaining significantly not reduced at early times - the NES absorber efficiently mitigates time response of both structural modes 1 and 2 (blue curves) by comparison with their free response without any absorber link. (red curves).

Scenario $C_{1} \leq S_{1} ; C_{2} \geq S_{2}$ (Fig. 3) is clearly recognized when noticing the decaying behavior of modal energies $E_{1}$ and $E_{2}$ defined by $E_{i}^{2}=\frac{\dot{y}_{i}^{2}}{\omega_{i}^{2}}+y_{i}^{2}$ with $i=1,2$ as shown in Fig. 9.b and 9.d.

$\mathrm{TET}_{2}$ mechanism is triggered at early time with a fastly decaying energy $E_{2}$ of mode 2 as witnessed in Fig. 9.d while at the same time, TET $_{1}$ is waiting for a delayed trigger and mode 1 dissipation is mainly due to its natural damping (see Fig. 9.b). As soon as modal energy $E_{2}$ pass downward its activation threshold $S_{2}$ at time $t \approx 2 s, \mathrm{TET}_{1}$ mechanism is to its turn triggered until $t \approx 17 \mathrm{~s}$ with a quasi-linear decaying trend. Beyond times $t \geq 17 \mathrm{~s}$, both competitive energy pumping mechanisms $\mathrm{TET}_{1}$ and $\mathrm{TET}_{2}$ give up being active. A wavelet time-frequency analysis of modal $y_{1}, y_{2}$ and NES $z$ responses gathered in Fig 10.a is additionally achieved (see Figs. 10.b though 10.d) to track changes into the instantaneous frequency content of previous responses. Amplitude $y_{2}$ of modal frequency $f_{2}=4.53 \mathrm{~Hz}$ soon decreases with an active TET Techanism $_{2}$ 


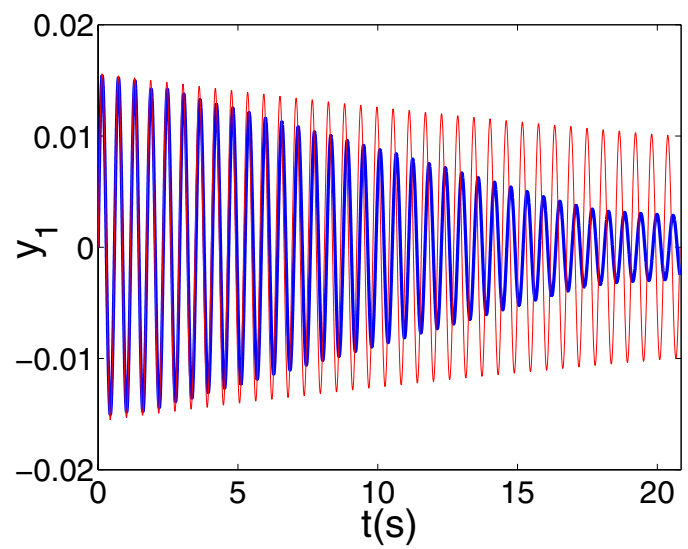

a) modal amplitude $y_{1}$ versus time

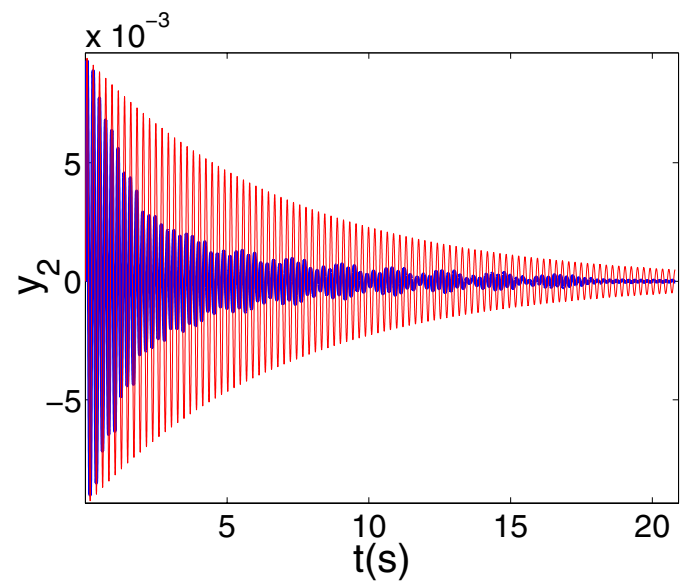

c) modal amplitude $y_{2}$ versus time

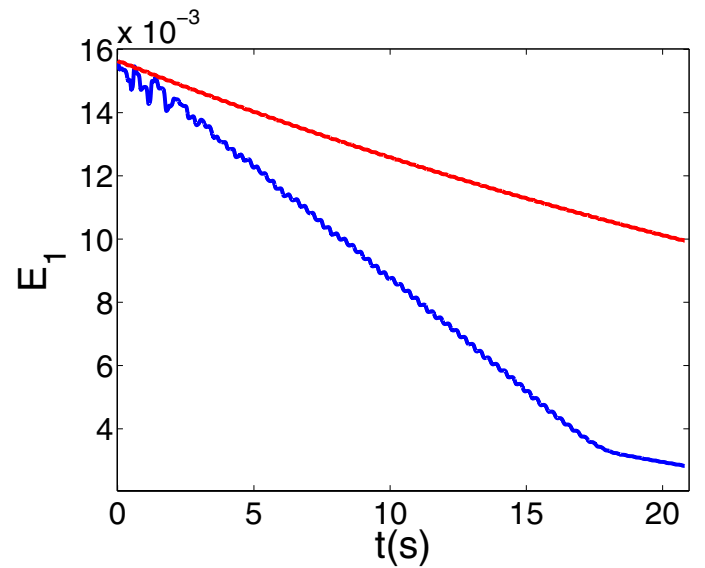

b) modal energy $E_{1}$ versus time

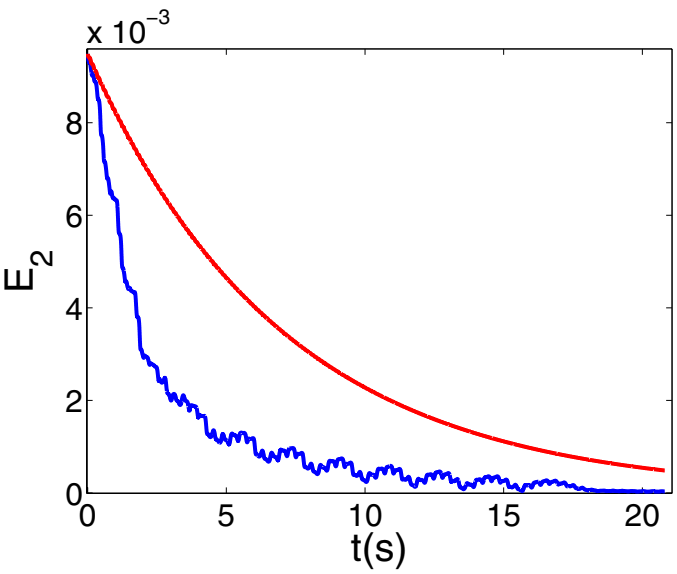

d) modal energy $E_{2}$ versus time

Figure 9: Mitigation of free oscillation response of modal quantities $\left(y_{i}, E_{i}\right)_{i=1,2}$ with $E_{i}^{2}=\frac{\dot{y}_{i}^{2}}{\omega_{i}^{2}}+y_{i}^{2}$ by using a NES (blue) and without any absorber (red).

until times $t \approx 2 s$ as seen in Fig. 10.d. In Fig. 10.c, modal amplitude $y_{1}$ related to frequency $f_{1}=1.73 \mathrm{~Hz}$ is continuously fed with energy until $\mathrm{TET}_{2}$ mobilizing mode 2 vanishes then $\mathrm{TET}_{1}$ mechanism is triggered and $y_{1}$ decay speed is increased after $t \approx 2 s$. It is also noticeable in Fig. 10.b that frequencies mobilized by the NES response are both modal frequencies $f_{1}$ and $f_{2}$. Amplitude $z$ is first resonating at the vicinity of modal frequency $f_{2}$ at early times when $\mathrm{TET}_{2}$ is active and then it is solely driven by frequency $f_{1}$ for later times as forecast by competitive targeted energy transfer scenario $C_{1} \leq S_{1} ; C_{2} \geq S_{2}$.

\subsection{Dynamic behavior with a pulse-like excitation}

A more complex transient regime is investigated in which both modes 1 and 2 initially at rest are excited with a gaussian pulse-like forcing vibrating respectively at the vicinity of frequencies $f_{1}=1.73 \mathrm{~Hz}$ and $f_{2}=4.53 \mathrm{~Hz}$. Equations of motions are hence given by: 


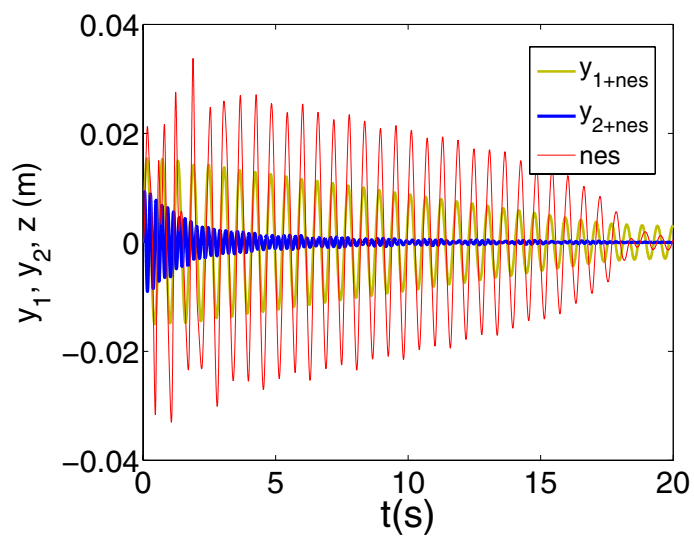

a) modal displacements $y_{1}, y_{2}, z$ versus time Energy Cuttof (\%) 22 Num. of contours 16 Contour Rirge v

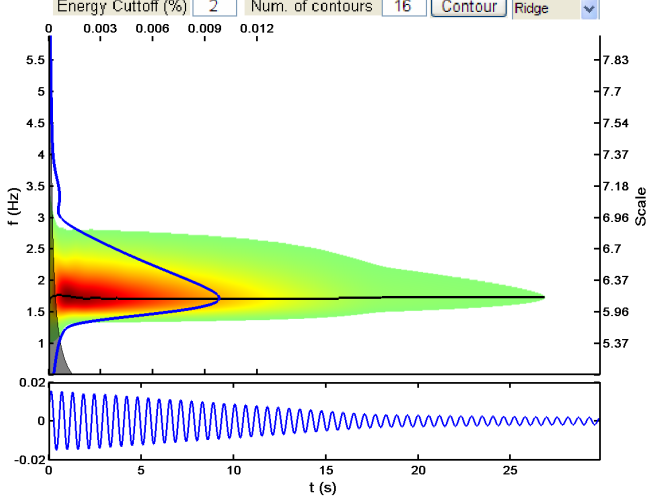

c) scalogram of $y_{1}$

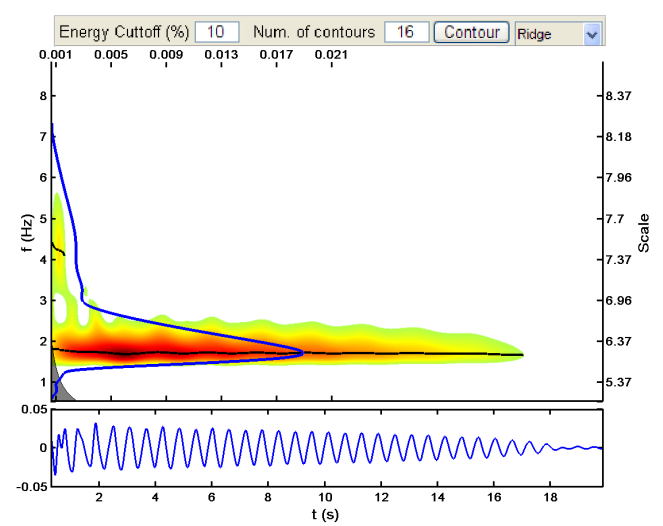

b) scalogram of $z$

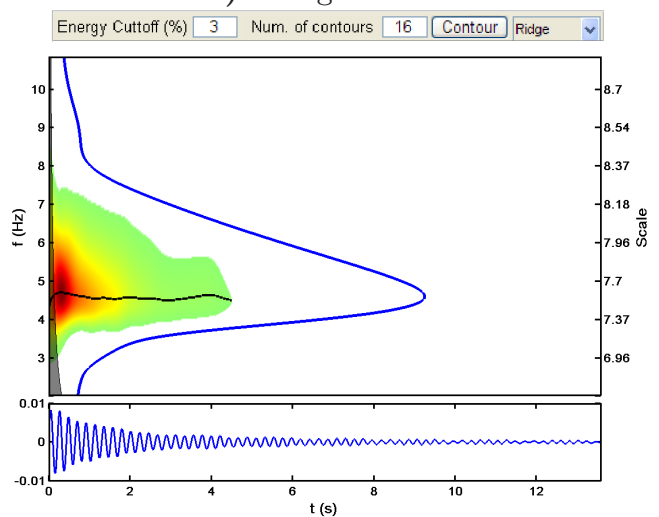

d) scalogram of $y_{2}$

Figure 10: Free oscillation response of modal displacements $y_{1}, y_{2}, z$ and their related wavelet time-frequency scalogram.

$$
\begin{cases}\ddot{y}_{1}+\epsilon_{1} \lambda_{1} \dot{y}_{1}+\omega_{1}^{2} y_{1}+\epsilon_{1} \lambda\left(\dot{y}_{1}+\dot{y}_{2}-\dot{z}\right)+\epsilon_{1} K\left(y_{1}+y_{2}-z\right)^{3} & =A_{1} \exp ^{-\left(t-t_{c}\right)^{2} / 2} \cos \left(\omega_{1}\left(t-t_{c}\right)\right) \\ \ddot{y}_{2}+\epsilon_{2} \lambda_{2} \dot{y}_{1}+\omega_{2}^{2} y_{2}+\epsilon_{2} \lambda\left(\dot{y}_{1}+\dot{y}_{2}-\dot{z}\right)+\epsilon_{2} K\left(y_{1}+y_{2}-z\right)^{3} & =A_{2} \exp ^{-\left(t-t_{c}\right)^{2} / 2} \cos \left(\omega_{2}\left(t-t_{c}\right)\right) \\ \ddot{z}+\lambda\left(\dot{z}-\dot{y}_{1}-\dot{y}_{2}\right)+K\left(z-y_{1}-y_{2}\right)^{3} & =0\end{cases}
$$

where $t_{c} \approx 5 \mathrm{~s}$ stands for a critical time chose such that quantity $\exp \left(t_{c}^{2} / 2\right)$ is almost vanishing.

Pulse amplitudes are chosen like:

- $A_{1}=0.135 \mathrm{~m} / \mathrm{s}^{2}$

- $A_{2}=0.270 \mathrm{~m} / \mathrm{s}^{2}$

and following trivial initial conditions

- $y_{1}(0)=0 \mathrm{~m}, y_{1}^{\prime}(0)=0 \mathrm{~m} / \mathrm{s}$ 


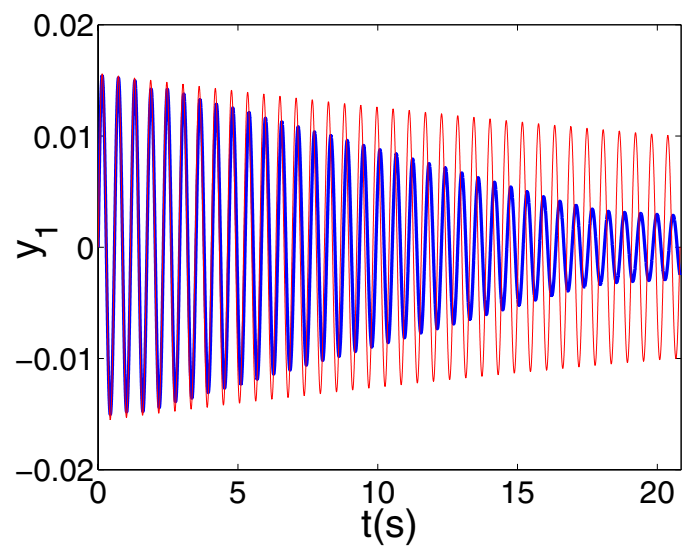

a) modal amplitude $y_{1}$ versus time

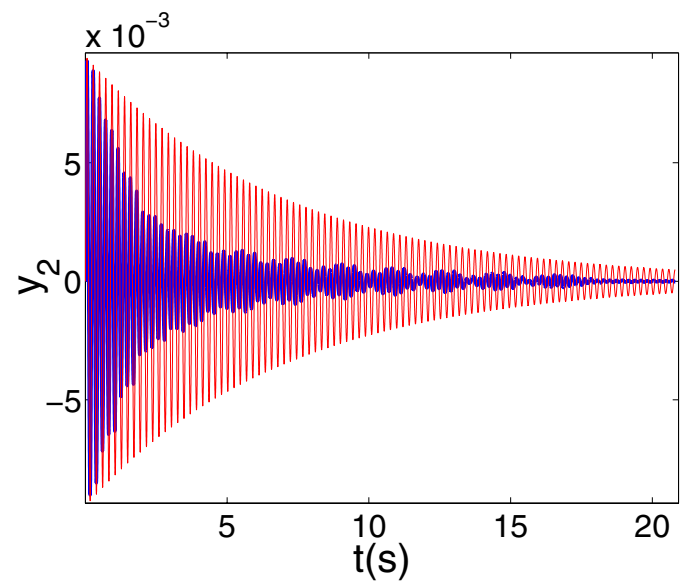

c) modal amplitude $y_{2}$ versus time

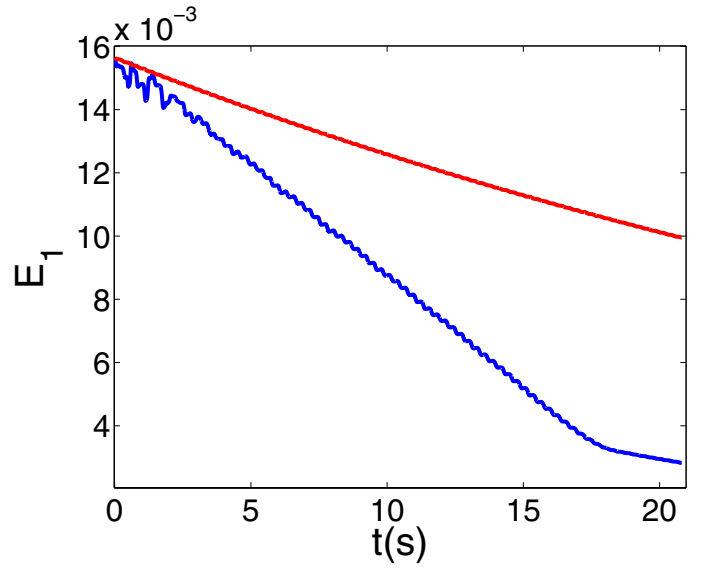

b) modal energy $E_{1}$ versus time

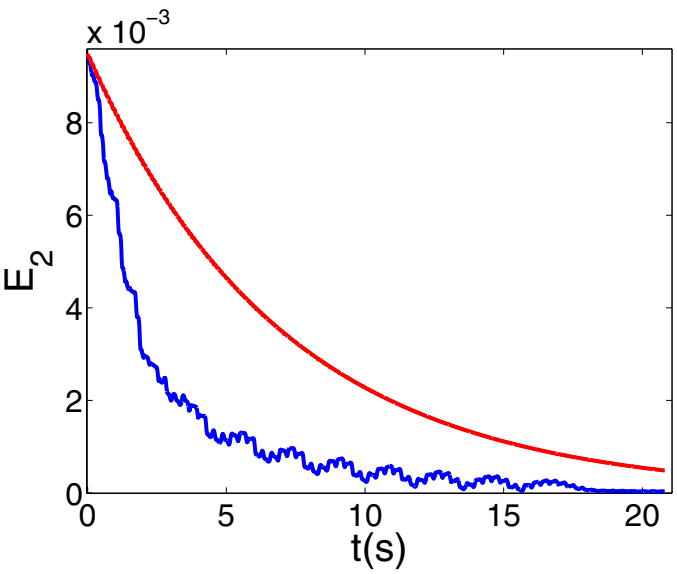

d) modal energy $E_{2}$ versus time

Figure 11: Attenuation of modal quantities $\left(y_{i}, E_{i}\right)_{i=1,2}$ with $E_{i}^{2}=\frac{\dot{y}_{i}^{2}}{\omega_{i}^{2}}+y_{i}^{2}$ excited by a pulse-like excitation by using a NES (blue) and without any absorber (red).

- $y_{2}(0)=0 \mathrm{~m}, y_{2}^{\prime}(0)=0 \mathrm{~m} / \mathrm{s}$

- $z(0)=0 \mathrm{~m}, z^{\prime}(0)=0 \mathrm{~m} / \mathrm{s}$

are considered for modal displacements and velocities in numerical simulations.

The dynamic behavior of modal amplitude responses $y_{1}$ and $y_{2}$ and related energies $E_{1}$ and $E_{2}$ displayed in Figs 11.a through 11.d is similar with the one studied in the case of free vibrations. TET $_{2}$ mechanism is triggered first thus fastly attenuating amplitude $y_{2}$ while $\mathrm{TET}_{1}$ remains inactive. Then $\mathrm{TET}_{1}$ becomes active for later times and breaks the slope of energy decay $E_{1}$ as shown in Fig.11.b. When both TET 1 and $\mathrm{TET}_{2}$ are disabled, energies $E_{1}$ and $E_{2}$ decay mainly due to the occurrence of modal damping effects. Similar explanation hold for related scalograms introduced in Figs 12.a though 12.d. 


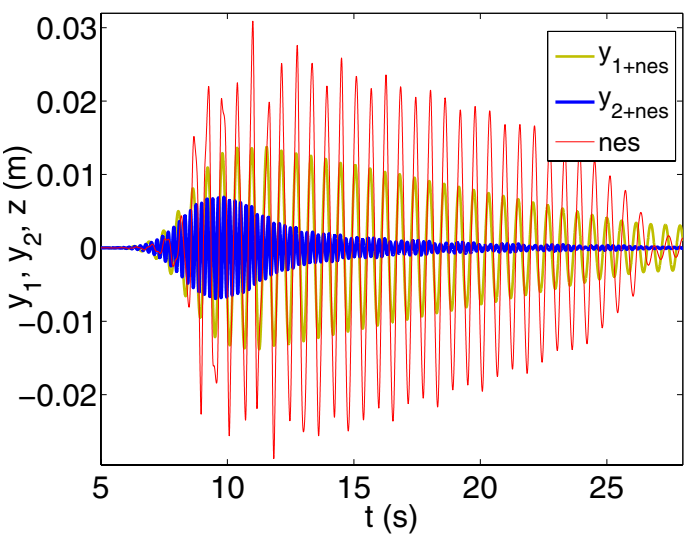

a) modal displacements $y_{1}, y_{2}, z$ versus time

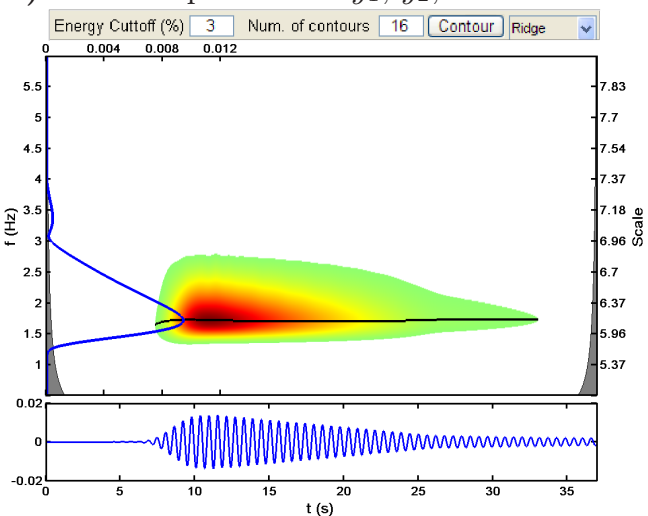

c) scalogram of $y_{1}$

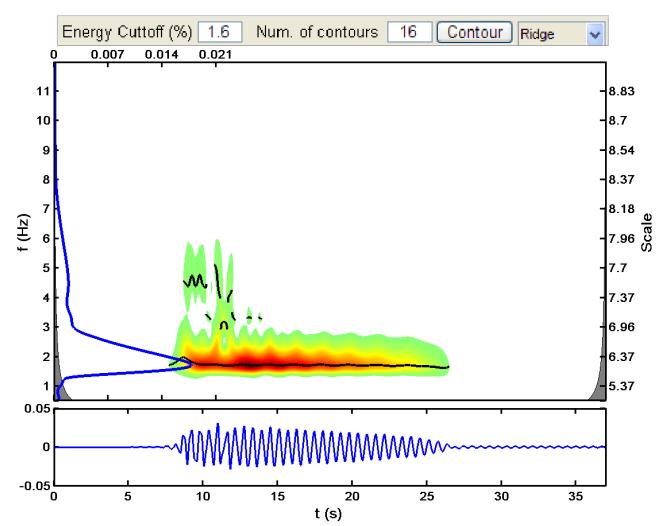

b) scalogram of $z$

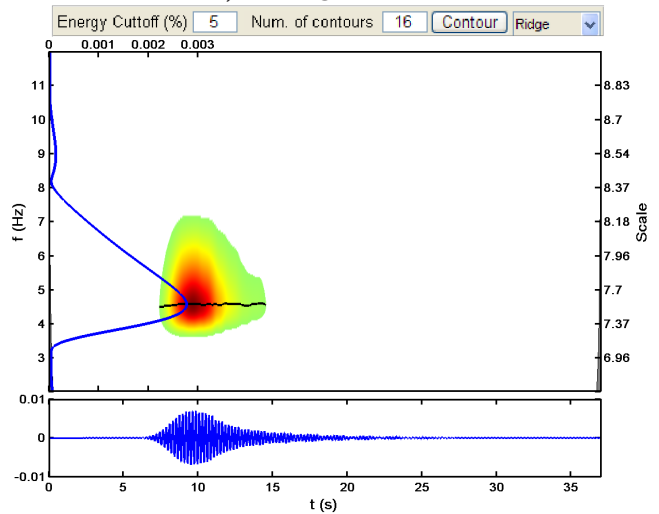

d) scalogram of $y_{2}$

Figure 12: Response of modal displacements $y_{1}, y_{2}, z$ to a pulse-like excitation and their related wavelet time-frequency scalogram.

\section{Conclusion}

The transient dynamic of a two degree-of-freedom system linked to a nonlinear energy sink by means of a essential nonlinear stiffness has been investigated analytically by using an original asymptotic expansion based on Manevitch's complexification method and featuring two small independent scales. The analytical study naturally exhibited two activation energy thresholds for targeted energy transfer likely to develop. Scenarios were also introduced to a priori forecast which and how competitive TET mechanisms are triggered knowing initial energies. An academic instance involving a two-storey building model was numerically simulated for comparison with analytical trends. Numerical results are in good agreement with expected analytical behaviors and thus validate TET scenarios. Futur prospect are now focusing on design methodologies to optimize energy pumping efficiency in the case of multiple TET mechanisms in competition. 


\section{Acknowledgment}

This work has been supported by French National Research Agency under contract ANR-07-BLAN-0193.

\section{References}

[1] Vakakis A. (2001), Inducing Passive Nonlinear Energy Sinks in Vibrating Systems, ASME Transactions, Journal of Sound and Vibration, 11, pp. 79-93.

[2] Gendelman O., Manevitch L., Vakakis A. and Closkey R. (2001), Energy Pumping in Nonlinear Mechanical Oscillators: Part I-Dynamics of the Underlying Hamiltonian Systems, Journal of Applied Mechanics, 68, pp. 34-41.

[3] Vakakis A. and Gendelman O. (2001), Energy Pumping in Non-linear Mechanical Oscillators: Part II-Resonance Capture, Journal of Applied Machanics, 68, pp. 42-48.

[4] Gendelman O. (2001), Transition of Energy to a Nonlinear Localized Mode in a highly Asysmmetric System of Two Oscillators, Nonlinear Dynamics, 25, pp. 237-253.

[5] Manevitch L. (2001), The description of localized normal modes in a chain of nonlinear coupled oscillators using complex variables, Nonlinear Dynamics, 25, pp. 95-109.

[6] Vakakis A., Kounadis A. and Raftoyiannis I. (1999), Use of nonlinear localization for isolating structures from earthquakeinduced motions, Earthquake Engineering and Structural Dynamics, 28, pp. 21-36.

[7] McFarland D., Gipson D., Vakakis A. and Bergman L. (2002), Characterization of an essentially nonlinear 2-DOF vibration test apparatus, 15th ASCE Engineering Mechanics Conference, Columbia University, New York, NY. Proceedings.

[8] McFarland D., Vakakis A. and Bergman L. (2002), Experimental verification of the performance of a non linear energy sink, 15th ASCE Engineering Mechanics Conference, Columbia University, New York, NY. Proceedings.

[9] McFarland D., Bergman L., Vakakis A., Manevitch L. and Gendelman O. (2002), Energy pumping into passive nonlinear energy sinks attached to forced linear substructures: analytical and experimental results, Ninth Conference on Nonlinear Vibration, Stability, and Dynamics of Structure, Session 3, Virginia Polytechnic Institute and State University.

[10] McFarland D., Bergman L. and Vakakis A. (2005), Experimantal study of non-linear energy pumping occurring at a single fast frequency, International Journal of Non-linear Mechanics, 40, pp. 891-899.

[11] Pernot S., Gloeckner M., Gourdon E., Lamarque C.H. and Griessman T. (2005), Experimental dynamics of a four storey building coupled with a nonlinear energy sink, EUROMECH ENOC'05 Conference, Eindhoven, The Netherlands.

[12] Pernot S., Gourdon E. and Lamarque C.H. (2006), Optimal design and multi-modal control with passive nonlinear absorber(s), 2nd International Conference on Nonlinear Normal Modes and Localization in Vibrating Systems, Samos, June 19-23th, Greece.

[13] Gourdon E., Taylor C.A., Alexander N.A., Lamarque C.H. and Pernot S. (2007), Experimental Verification of Nonlinear Energy pumping under transient forcing with strongly nonlinear coupling, Journal of sound and vibration, 300, pp. 522-551.

[14] Gourdon E., Lamarque C.H. and Pernot S. (2007), Contribution to efficiency of irreversible passive energy pumping with a strong nonlinear attachment, Nonlinear Dynamics, DOI: 10.1007/s11071-007-9229-y.

[15] Gourdon E., Pernot S. and Lamarque C.H. (2007), Energy pumping with multiple passive nonlinear absorbers, Euromech Colloquium 483: Geometrically nonlinear vibrations of structures, FEUP, Porto, 9-11 July 2007, Portugal.

[16] Gendelman O., Manevitch L., Vakakis A. and Bergman L. (2003), A Degenerate Bifurcation Structure in the Dynamics of Coupled Oscillators with Essential Stiffness Nonlinearities, Nonlinear Dynamics, 33, pp. 1-10.

[17] Vakakis A., Manevitch L., Gendelman O. and Bergman L. (2003), Dynamics og linear discrete systems connected to local, essentially non-linear attachements, Journal of Sound and Vibration, 264, pp. 559-577. 
[18] Manevitch L., Gendelman O., Musienko A., Vakakis A. and Bergman L. (2003), Dynamics Interaction of a Semi-infinite Linear Chain of Coupled Oscillators with a Strongly Nonlinear Attachment, Physica D, 25, pp. 1-18.

[19] Vakakis A. (2003), Shock Isolation Trough the Use of Nonlinear Energy Sinks, Journal of Sound and Vibration and Control, 9, pp. 79-93.

[20] Vakakis A., Manevitch L., Musienko A, Kerschen G. and Bergman L. (2004), Transient dynamics of a dispersive elastic wave guide weakly coupled to an essentially nonlinear end attachement, Wave motion, 41, pp. 109-132.

[21] Vakakis A., FcFarland D., Bergman L., Manevitch L. and Gendelman O. (2004), Isolated resonance captures and resonance capture cascades leading to single or multi-mode passive energy pumping in damped coupled oscillators, Journal of Vibration and Acoustics, 126, pp. 235-244.

[22] Gendelman O. (2004), Bifurcations of Nonlinear Normal Modes of Linear Oscillator with Strongly Nonlinear damped Attachement, Nonlinear Dynamics, 37(2), pp. 115-128.

[23] Gendelman O., Gorlov D., Manewitch L. et Moussienko A. (2005), Dynamics of coupled linear and essentially nonlinear oscillators with substantially different masses, Journal of Sound and Vibration, 286(1-2), pp. 1-19.

[24] Gendelman O. and Lamarque C.-H. (2005), Dynamics of linear oscillator coupled to strongly nonlinear attachement with multiple states of equilibrium, Chaos, Solitons and Fractals, 24, pp. 501-509.

[25] Manevitch L., Musienco A. and Lamarque C.-H. (2006), New analytical approach to energy pumping problem in strongly nonhomogeneous 2dof systems, Mecanica, ISSN 0025-6455 (Print) 1572-9648 (Online), DOI 10.1007/s11012-006-9021-y, pp. $77-83$.

[26] Gendelman O., Gourdon E., et Lamarque C.-H. (2006), Quasiperiodic Energy Pumping in Coupled Oscillators under Periodic Forcing, Journal of Soud and Vibration, 294, pp. 651-662.

[27] Gendelman O. and Starosvetsky Y. (2007), Quasiperiodic reponse regimes of strongly nonlinear vibration absorber, Journal of Applied Mechanics, vol 74 (2), pp. 325-331.

[28] O.V. Gendelman (2008), Targeted energy transfer in systems with non-polynomial nonlinearity. Journal of Sound and Vibration, vol.315 (3), pp. 732-745.

[29] Rosenberg, R. (1961), On normal vibrations of a general class of nonlinear dual-mode systems. Journal of Applied Mechanics, $28: 275-283$.

[30] Gourdon E., Contrôle passif de vibrations par pompage énergétique. PhD Thesis, Ecole doctorale MEGA, Génie mécanique, Ecole Centrale de Lyon et ENTPE, 277 p., 2006. (In french). 